\title{
Auxiliary master equation approach to nonequilibrium correlated impurities
}

\author{
Antonius Dorda, Martin Nuss, Wolfgang von der Linden, and Enrico Arrigoni \\ Institute of Theoretical and Computational Physics, Graz University of Technology, 8010 Graz, Austria
}

(Received 23 December 2013; published 7 April 2014)

\begin{abstract}
We present a numerical method for the study of correlated quantum impurity problems out of equilibrium, which is particularly suited to address steady-state properties within dynamical mean field theory. The approach, recently introduced by Arrigoni et al. [Phys. Rev. Lett. 110, 086403 (2013)], is based upon a mapping of the original impurity problem onto an auxiliary open quantum system, consisting of the interacting impurity coupled to bath sites as well as to a Markovian environment. The dynamics of the auxiliary system is governed by a Lindblad master equation whose parameters are used to optimize the mapping. The accuracy of the results can be readily estimated and systematically improved by increasing the number of auxiliary bath sites, or by introducing a linear correction. Here, we focus on a detailed discussion of the proposed approach including technical remarks. To solve for the Green's functions of the auxiliary impurity problem, a non-Hermitian Lanczos diagonalization is applied. As a benchmark, results for the steady-state current-voltage characteristics of the single-impurity Anderson model are presented. Furthermore, the bias dependence of the single-particle spectral function and the splitting of the Kondo resonance are discussed. In its present form, the method is fast, efficient, and features a controlled accuracy.
\end{abstract}

DOI: 10.1103/PhysRevB.89.165105

PACS number(s): 71.15.-m, 71.27.+a, 73.63.Kv, 73.23.-b

\section{INTRODUCTION}

Correlated systems out of equilibrium have recently attracted increasing interest due to the significant progress in a number of related experimental fields. Advances in microscopic control and manipulation of quantum mechanical many-body systems within quantum optics [1] and ultracold quantum gases, for example in optical lattices [2-6], have long reached high accuracy and versatility. Ultrafast laser spectroscopy $[7,8]$ offers the possibility to explore and understand electronic dynamics in unprecedented detail. Experiments in condensed matter nanotechnology [9], spintronics [10], molecular junctions [11-16], and quantum wires or quantum dots $[17,18]$ are able to reveal effects of the interference of few microscopic quantum states. The nonequilibrium nature of such experiments does not only offer a new route to explore fundamental aspects of quantum physics, such as nonequilibrium quantum phase transitions [19], the interplay between quantum entanglement, dissipation, and decoherence [20], or the pathway to thermalization [21,22], but also suggests the possibility of exciting future applications $[11,23]$.

Addressing the dynamics of correlated quantum systems poses a major challenge to theoretical endeavors. In this respect, quantum impurity models help improving our understanding of fermionic many-body systems. In particular, the single-impurity Anderson model (SIAM) [24], which was originally devised to study magnetic impurities in metallic hosts $[25,26]$, has become an important tool in many areas of condensed matter physics [27,28]. Most prominently, it features nonperturbative many-body physics which manifest

\footnotetext{
*dorda@tugraz.at

Published by the American Physical Society under the terms of the Creative Commons Attribution 3.0 License. Further distribution of this work must maintain attribution to the author(s) and the published article's title, journal citation, and DOI.
}

in the Kondo effect [29]. It provides the backbone for all calculations within dynamical mean field theory (DMFT) $[28,30]$, a technique which allows us to understand the properties of a broad range of correlated systems and becomes exact in the limit of infinite dimensions [31]. The basic physical properties of the SIAM in equilibrium are quite well understood [29] thanks to the pioneering work from Kondo [32], renormalization group [33], as well as perturbation theory (PT) [34-37] and the mapping to its low-energy realization, the Kondo model [38].

The SIAM out of equilibrium provides a description for several physical processes such as, for example, nonlinear transport through quantum dots $[17,39]$, correlated molecules $[13,14,40-42]$, or the influence of adsorbed atoms on surfaces or bulk transport [43]. As in the equilibrium case, the solution of the SIAM constitutes the bottleneck of nonequilibrium DMFT [44-51] calculations. Therefore, accurate and efficient methods to obtain dynamical correlation functions of impurity models out of equilibrium are required in order to describe time-resolved experiments on strongly correlated compounds $[7,8]$ and to understand their steady-state transport characteristics [23].

However, nonequilibrium correlated impurity models still pose an exciting challenge to theory. Our work addresses this issue with special emphasis on the steady state. But, before introducing this work in Sec. I, we briefly review previous approaches. In recent times, a number of computational techniques have been devised to handle the SIAM out of equilibrium. Among them are scattering-state Bethe ansatz (BA) [52], scattering-state NRG (SNRG) [53-55], noncrossing approximation studies [56,57], fourth-order Keldysh PT [58], other perturbative methods $[59,60]$ in combination with the renormalization group (RG) [61-65], iterative summation of real-time path integrals [66], time-dependent NRG [67], flow equation techniques $[68,69]$, the time-dependent density matrix RG (DMRG) [70-75] applied to the SIAM [76,77], nonequilibrium cluster PT (CPT) [78], the nonequilibrium variational cluster approach (VCA) [79,80], dual fermions 
[81], the functional RG (fRG) [82,83], diagrammatic quantum Monte Carlo (QMC) [84,85], continuous time QMC (CTQMC) calculations on an auxiliary system with an imaginary bias [86-90], superoperator techniques [91,92], many-body PT and time-dependent density functional theory [93], generalized slave-boson methods [94], real-time RG (rtRG) [95], time-dependent Gutzwiller mean field calculations [96], and generalized master equation approaches [97]. Comparisons of the results of some of these methods are available in literature [77,98,99] and time scales have been discussed in Ref. [100].

Despite this large number of approaches, only a limited number of them is applicable to nonequilibrium DMFT, and very few are still accurate for large times in steady state. Beyond the quadratic action for the Falicof-Kimball model [46,101,102], iterated PT (IPT) [45], numerical renormalization group (NRG) [48], real-time QMC [48,103], the noncrossing approximation (NCA) [104,105], and recently Hamiltonian-based impurity solvers [106] have been applied in the time-dependent case. Some of the above approaches, such as QMC [49] and DMRG [73], are very accurate in addressing the short- and medium-time dynamics, but in some cases the accuracy decreases at long times and a steady state can not be reliably identified. Some other methods are perturbative and/or valid only in certain parameter regions or for restricted models. RG approaches (e.g., [61]) are certainly more appropriate to identify the low-energy behavior.

\section{This work}

In this paper, we discuss a method, first proposed in [51], which addresses the correlated impurity problem out of equilibrium, and is particularly efficient for the steady state. The accuracy of the results is controlled as it can be directly estimated by analyzing the bath hybridization function (details following). Here, we extend, test, and provide details of this approach and its implementation. The basic idea is to map the impurity problem onto an auxiliary open system, consisting of a small number of bath sites coupled to the interacting impurity and, additionally, to a so-called Markovian environment [107]. The parameters of this auxiliary open quantum system are obtained by optimization in order to represent the original impurity problem as accurately as possible. The auxiliary system dynamics are governed by a Lindblad master equation which is solved exactly with the non-Hermitian Lanczos method. The crucial point is that the overall accuracy of the method is thus solely determined by how well the auxiliary system reproduces the original one. This can be, in principle, improved by increasing the number of auxiliary bath sites.

In this study, we provide convincing benchmarks for the steady-state properties of the SIAM coupled to two metallic leads under bias voltage. We include a discussion of convergence as a function of the number of bath sites and present a scheme to estimate the error and partially correct for it. In its presented form, the method is fast, efficient, and is directly applicable to steady-state dynamical mean field theory [51] for which previously suggested methods are less reliable. Extending the method to treat time-dependent properties and multiorbital systems is possible, in principle, however with a much heavier computational effort.
The paper is organized as follows: In Sec. II A, the SIAM under bias voltage is introduced. In Sec. II B, we introduce nonequilibrium Green's functions and in Secs. II C and II D, we outline the auxiliary master equation approach where we also focus on details of our particular implementation. Results for the steady state, including the equilibrium situation, are presented in Sec. III. This includes the steady-state currentvoltage characteristics which we compare with exact results from matrix product state (MPS) time evolution [77] as well as data for the spectral function under bias which we compare with nonequilibrium NRG [54]. We conclude and give an outlook in Sec. IV.

\section{AUXILIARY MASTER EQUATION APPROACH}

As discussed above, the method is particularly suited to deal with nonequilibrium steady-state properties caused by different temperatures and/or chemical potential in the leads of a correlated quantum impurity system. As such, it can be readily used as impurity solver for nonequilibrium DMFT [46,51]. Here, we illustrate its application to the fermionic SIAM with two leads having different chemical potentials, and, in principle, different temperatures.

\section{A. Nonequilibrium single-impurity Anderson model}

We consider a single Anderson impurity coupled to electronic leads under bias voltage [see Fig. 1(a)]

$$
\hat{\mathcal{H}}=\hat{\mathcal{H}}_{\text {imp }}+\hat{\mathcal{H}}_{\text {res }}+\hat{\mathcal{H}}_{\text {coup }}
$$

The impurity orbital features charge as well as spin degrees of freedom and is subject to a local Coulomb repulsion $U$ :

$$
\hat{\mathcal{H}}_{\text {imp }}=\epsilon_{f} \sum_{\sigma} f_{\sigma}^{\dagger} f_{\sigma}+U \hat{n}_{\uparrow}^{f} \hat{n}_{\downarrow}^{f} .
$$
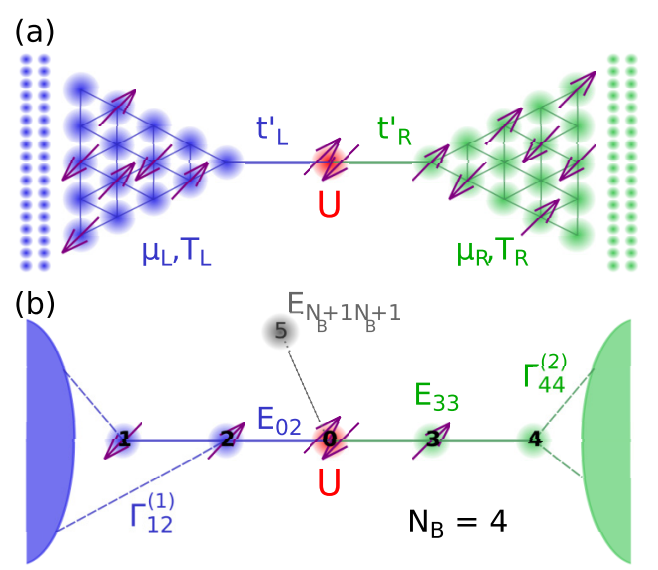

FIG. 1. (Color online) (a) Sketch of the quantum impurity model (1) consisting of an impurity with interaction $U$ coupled via hybridizations $t_{\lambda}^{\prime}$ to noninteracting leads at chemical potential $\mu_{\lambda}$ and temperature $T_{\lambda}, \lambda \in\{L, R\}$. (b) Illustration of the auxiliary open quantum system [Eq. (10a)] with single-particle parameters $E_{\mu \nu}$ and Lindblad dissipators $\Gamma_{\mu \nu}^{\kappa}$ consisting of the impurity at site $f=0, N_{B}$ bath sites $\left(N_{B}=4\right.$ in the plot), as well as a Markovian environment (shaded areas). When evaluating linear corrections (see Appendix C), an additional site $N_{B}+1$ is used. 
Here, $f_{\sigma}^{\dagger} / f_{\sigma}$ denote fermionic creation/annihilation operators for the impurity orbital with spin $\sigma \in\{\uparrow, \downarrow\}$, respectively. The particle-number operator is defined in the usual way $\hat{n}_{\sigma}^{f}=$ $f_{\sigma}^{\dagger} f_{\sigma}$ and the impurity onsite potential is $\epsilon_{f}=\left(V_{G}-\frac{U}{2}\right)$, with gate voltage $V_{G}=0$ at particle-hole symmetry. The impurity is coupled to two noninteracting electronic leads $\lambda \in\{L, R\}$ with dispersion $\epsilon_{\lambda k}$ :

$$
\hat{\mathcal{H}}_{\mathrm{res}}=\sum_{\lambda k \sigma}\left(\epsilon_{\lambda}+\epsilon_{\lambda k}\right) c_{k \lambda \sigma}^{\dagger} c_{k \lambda \sigma} .
$$

The effect of a bias voltage $\phi$ is to shift the chemical potential and the onsite energies of the two leads by $\epsilon_{\lambda}= \pm \frac{\phi}{2}$, respectively. For the energies $\epsilon_{\lambda k}$ of the leads we will consider two cases.

(i) Two tight-binding semi-infinite chains with nearestneighbor hopping $t$, corresponding to a semicircular electronic density of states (DOS): In this case, the boundary retarded single-particle Green's function of the two uncoupled leads is given by [108-110]

$$
g_{\lambda}^{R}(\omega)=g_{\mathrm{SC}, \lambda}^{R}(\omega)=\frac{\omega-\epsilon_{\lambda}}{2 t^{2}}-i \frac{\sqrt{4 t^{2}-\left(\omega-\epsilon_{\lambda}\right)^{2}}}{2 t^{2}},
$$

with a bandwidth of $D_{\mathrm{SC}}=4 t$.

(ii) A constant DOS with a bandwidth $D_{\mathrm{WB}}=\pi t$ results in boundary Green's functions [109]

$$
g_{\lambda}^{R}(\omega)=g_{\mathrm{WB}, \lambda}^{R}(\omega)=-\frac{1}{D_{\mathrm{WB}}} \ln \left(\frac{\omega-\epsilon_{\lambda}-\frac{D_{\mathrm{WB}}}{2}}{\omega-\epsilon_{\lambda}+\frac{D_{\mathrm{WB}}}{2}}\right) .
$$

The choice $D_{\mathrm{WB}}=\pi t$ makes sure that the DOS at $\omega=0$ of both lead types coincide. The leads are coupled to the impurity orbital by

$$
\hat{\mathcal{H}}_{\text {coup }}=\sum_{\lambda \sigma} t_{\lambda}^{\prime} \frac{1}{\sqrt{N_{k}}} \sum_{k}\left(c_{k \lambda \sigma}^{\dagger} f_{\sigma}+f_{\sigma}^{\dagger} c_{k \lambda \sigma}\right),
$$

where we take the same hybridization $t_{\lambda}^{\prime}=-0.3162 t$ for both leads, and $N_{k} \rightarrow \infty$ is the number of $k$ points. Expressions presented below are valid for arbitrary temperatures, although we will show results for zero temperature only, which is numerically the most unfavorable case [111]. The setup chosen here represents by no means a limitation of the method and extensions to more complicated situations, such as nonsymmetric couplings, off particle-hole symmetry, etc., are straightforward.

\section{B. Steady-state nonequilibrium Green's functions}

We are interested in the steady-state behavior under bias voltage of the model described by Eq. (1). We assume that such a steady state exists and is unique [112]. We denote the singleparticle Green's function of the impurity in the nonequilibrium Green's function (Keldysh) formalism by [113-117]

$$
\underline{G}(\omega)=\left(\begin{array}{cc}
G^{R}(\omega) & G^{K}(\omega) \\
0 & G^{A}(\omega)
\end{array}\right) .
$$

Fourier transformation to energy $\omega$ is possible since in the steady state the system becomes time translationally invariant.
In that case, the memory of the initial condition has been fully washed away, so there is no contribution from the Matsubara branch [118]. We will use an underline ... to denote two-point functions with the Keldysh matrix structure as in Eq. (4).

The Green's function of the correlated impurity can be expressed via Dyson's equation

$$
\underline{G}^{-1}(\omega)=\underline{G}_{0}^{-1}(\omega)-\underline{\Sigma}(\omega),
$$

where $\underline{\Sigma}(\omega)$ is the impurity self-energy. The noninteracting impurity Green's function $\underline{G}_{0}(\omega)$ can be written in the form

$$
\underline{G}_{0}^{-1}(\omega)=\underline{g}_{0}^{-1}(\omega)-\underline{\Delta}(\omega),
$$

$\underline{g}_{0}(\omega)$ being the noninteracting Green's function of the disconnected impurity [108], and

$$
\underline{\Delta}(\omega)=\sum_{\lambda} t_{\lambda}^{\prime 2} \underline{g}_{\lambda}(\omega)
$$

is the hybridization function of the leads (a $2 \times 2$ Keldysh object, in contrast to the equilibrium case, where it is convenient to work in Matsubara space). We define an equilibrium Anderson width [29] for each lead $\Delta_{0} \equiv-\frac{1}{2} \operatorname{Im}\left[\underline{\Delta}^{R}(\omega=\right.$ $0)]=\frac{t_{\lambda}^{\prime 2}}{t} \approx 0.1 t$. In the following, we will use $\Delta_{0}$ as a unit of energy and in addition we choose $\hbar=e=1$.

The boundary Green's functions $\underline{g}_{\lambda}$ of each disconnected lead is determined by (a) its retarded component $g_{\lambda}^{R}$ [either Eqs. (2) or (3)], (b) its advanced component $g_{\lambda}^{A}=g_{\lambda}^{R *}$, and (c) its Keldysh component, which satisfies the fluctuation dissipation theorem

$$
g_{\lambda}^{K}(\omega)=2 i\left[1-2 p_{\mathrm{F}}\left(\omega-\mu_{\lambda}\right)\right] \operatorname{Im}\left[g_{\lambda}^{R}(\omega)\right]
$$

since the disconnected leads are in equilibrium. Here, $p_{\mathrm{F}}(\omega-$ $\left.\mu_{\lambda}\right)$ is the Fermi distribution with chemical potential $\mu_{\lambda}$. For the noninteracting isolated impurity, one can take $\left(g_{0}^{-1}\right)^{R}=$ $\omega-\epsilon_{f}$ and $\left(g_{0}^{-1}\right)^{K}=0$ since infinitesimals $0^{+}$can be neglected after coupling to the leads (unless there are bound states). As usual, the presence of the interaction $U$ makes the solution of the problem impurity plus leads a major challenge both in equilibrium as well as out of equilibrium, which we plan to address in this paper.

Similarly to the equilibrium case, the action of the leads on the impurity is completely determined by the hybridization function $\Delta(\omega)$, independently of how the leads are represented in detail. In other words, if one constructs a different configuration of leads (e.g., with more leads with different temperatures, DOS, etc.), which has the same $\Delta(\omega)$, i.e. the same $\Delta^{R}(\omega)$ and $\Delta^{K}(\omega)$ as Eq. (7), then the resulting local properties of the interacting impurity, e.g., the Green's function $\underline{G}(\omega)$ are the same. This holds provided the leads contain noninteracting fermions only.

The approach we suggested in Ref. [51] precisely exploits this property. The idea is to replace the impurity plus leads system [Eq. (1)] by an auxiliary one which reproduces $\underline{\Delta}(\omega)$ as accurately as possible, and at the same time can be solved exactly by numerical methods, such as Lanczos exact diagonalization. Details on the construction of the auxiliary impurity system are given in the following.

The self-energy $\underline{\Sigma}_{\mathrm{aux}}(\omega)$ of the auxiliary system, obtained by exact diagonalization, is used in analogy to DMFT [28,119] 
as an approximation to the physical self-energy of the original impurity system. Inserting $\underline{\Sigma}(\omega) \approx \underline{\Sigma}_{\text {aux }}(\omega)$ into Eqs. (5) and (6), together with the exact hybridization function $\underline{\Delta}(\omega)$ yields an approximation for the physical Green's function. From this, observables such as the current or the spectral function are then calculated. We emphasize that the accuracy of this approximation can be controlled by the difference between the $\underline{\Delta}_{\text {aux }}(\omega)$ of the auxiliary system and the physical one $\underline{\Delta}(\omega)$, and that this can be, in principle, systematically improved, as discussed below.

\section{Auxiliary open quantum system}

The idea presented here is strongly related to the exact diagonalization (ED) approach for the DMFT impurity problem in equilibrium $[28,119]$. Here, the infinite leads are replaced by a small number of bath sites, whose parameters are optimized by fitting the hybridization function in Matsubara space. The reduced system of bath sites plus impurity is then solved by Lanczos ED [120]. This approach can not be straightforwardly extended to the nonequilibrium steady-state case for several reasons: (i) since the small bath is finite, its time dependence is (quasi)periodic, i.e., no steady state is reached, (ii) there is no Matsubara representation out of equilibrium [121], thus, one is forced to use real energies but (iii) in this case $\operatorname{Im}\left[\Delta_{\text {aux }}^{R}(\omega)\right]$ of the small bath consists of $\delta$ peaks and can hardly be fitted to a smooth $\Delta^{R}(\omega)$. The solution we suggested in Ref. [51] consists in additionally coupling the small bath to a Markovian environment, which makes it effectively "infinitely large," and solves problems (i) and (iii) above. Specifically, we replace the impurity plus leads model [Eq. (1)] by an auxiliary open quantum system consisting of the impurity plus a small number of bath sites, which in turn are coupled to a Markovian environment.

The dynamics of the system (consisting of bath sites and impurity), including the effect of the Markovian environment is expressed in terms of the Lindblad quantum master equation which controls the time dependence of its reduced density operator $\hat{\rho}[107,122]$ :

$$
\dot{\hat{\rho}}=\hat{\hat{\mathcal{L}}} \hat{\rho} \text {. }
$$

The Lindblad superoperator [123]

$$
\hat{\hat{\mathcal{L}}}=\hat{\hat{\mathcal{L}}}_{H}+\hat{\hat{\mathcal{L}}}_{D}
$$

consists of a unitary contribution

$$
\hat{\hat{\mathcal{L}}}_{H} \hat{\rho}=-i\left[\hat{\mathcal{H}}_{\mathrm{aux}}, \hat{\rho}\right],
$$

as well as a nonunitary, dissipative term originating from the coupling to the Markovian environment

$$
\begin{aligned}
\hat{\mathcal{L}}_{D} \hat{\rho} \equiv & 2 \sum_{\mu \nu=0}^{N_{B}} \sum_{\sigma}\left[\Gamma_{\nu \mu}^{(1)}\left(d_{\mu \sigma} \hat{\rho} d_{\nu \sigma}^{\dagger}-\frac{1}{2}\left\{\hat{\rho}, d_{\nu \sigma}^{\dagger} d_{\mu \sigma}\right\}\right)\right. \\
& \left.+\Gamma_{\nu \mu}^{(2)}\left(d_{\nu \sigma}^{\dagger} \hat{\rho} d_{\mu \sigma}-\frac{1}{2}\left\{\hat{\rho}, d_{\mu \sigma} d_{\nu \sigma}^{\dagger}\right\}\right)\right]
\end{aligned}
$$

where $[\hat{A}, \hat{B}]$ and $\{\hat{A}, \hat{B}\}$ denote the commutator and anticommutator, respectively. The unitary time evolution is generated by the Hamiltonian

$$
\hat{\mathcal{H}}_{\mathrm{aux}}=\sum_{\mu \nu=0}^{N_{B}} \sum_{\sigma} E_{\mu \nu} d_{\mu \sigma}^{\dagger} d_{\nu \sigma}+U d_{f \uparrow}^{\dagger} d_{f \uparrow} d_{f \downarrow}^{\dagger} d_{f \downarrow},
$$

describing a fermionic "chain" ( $E_{\mu \nu}$ is nonzero only for onsite and nearest-neighbor terms). It is convenient to choose the interacting impurity at site $f=0$ and $N_{B}$ auxiliary bath sites at $\mu, v=1, \ldots, N_{B}$ (see Fig. 1(b)). As usual, $d_{\mu \sigma}^{\dagger} / d_{\mu \sigma}$ create/annihilate the corresponding auxiliary particles. The quadratic form of the dissipator [Eq. (10b)] corresponds to a noninteracting Markovian environment. The dissipation matrices $\Gamma_{\mu \nu}^{(\kappa)}, \kappa \in\{1,2\}$, are Hermitian and positive semidefinite [122]. The advantage of replacing the impurity problem by the auxiliary one described by Eqs. (9)-(11), is that for a small number of bath sites the dynamics of the interacting auxiliary system can be solved exactly by diagonalization of the superoperator $\hat{\hat{\mathcal{L}}}$ in the space of many-body density operators (see Sec. II D 2).

Intuitively, one can consider the effective system as a truncation of the original chain described by Eq. (1), whereby the Markovian environment compensates for the missing "pieces." However, this would still be a crude approximation and, in addition, it would not be clear how to introduce the chemical potential in the Markovian environment (except for weak coupling). Our strategy, similarly to the equilibrium case, consists in simply using the parameters of the auxiliary system in order to provide an optimal fit to the bath spectral function $\Delta(\omega)$. The parameters for the fit are, in principle, $E_{\mu \nu}$ and $\bar{\Gamma}_{\mu \nu}^{(\kappa)}$. However, one should consider that there is a certain redundancy. In other words, several combinations of parameters lead to the same $\underline{\Delta}(\omega)$. For example, it is well known in equilibrium that in the case of the $E_{\mu \nu}$ one can restrict to diagonal and nearest-neighbor terms only [124].

The accuracy of the results will be directly related to the accuracy of the fit to $\underline{\Delta}(\omega)$, and this is expected to increase rapidly with the number of fit parameters, which obviously increases with $N_{B}$. On the other hand, also the computational complexity necessary to exactly diagonalize the interacting auxiliary system increases exponentially with $N_{B}$. The fit does not present a major numerical difficulty, as the determination of the hybridization functions of both the original model [Eq. (7)], as well as the one of the auxiliary system $\underline{\Delta}_{\text {aux }}(\omega)$ described by the Lindblad equation (10) require the evaluation of $\underline{G}_{0}$ [cf. (6)], i.e., the solution of a noninteracting problem.

The fit is obtained by minimizing the cost function

$$
\begin{aligned}
\chi\left(E_{\mu \nu}, \Gamma_{\mu \nu}^{(\kappa)}\right)= & \sum_{\alpha \in\{R, K\}} \int_{-\infty}^{\infty} d \omega W^{\alpha}(\omega) \\
& \times\left|\Delta^{\alpha}(\omega)-\Delta_{\text {aux }}^{\alpha}\left(\omega ; E_{\mu \nu}, \Gamma_{\mu \nu}^{(\kappa)}\right)\right|^{n}
\end{aligned}
$$

with respect to the parameters of the auxiliary system. The advanced component does not need to be considered as $\Delta^{A}=\Delta^{R *}$. Of course, as in ED-based DMFT, there exists an ambiguity which is related to the choice of the weight function $W^{\alpha}(\omega)$, which also sets the integral boundaries. This uncertainty is clearly reduced upon increasing $N_{B}$.

Depending on the expected physics, it might be useful to adopt an energy-dependent weight function. This could be 
used, for example, to describe the physics around the chemical potentials more accurately.

Once the auxiliary system is defined in terms of $E_{\mu \nu}$ and $\Gamma_{\mu \nu}^{(\kappa)}$, the corresponding interacting nonequilibrium problem (10) can be solved by an exact diagonalization of the non-Hermitian superoperator $\hat{\hat{\mathcal{L}}}$ within the space of many-body density operators. The dimension of this space is equal to the square of the dimension of the many-body Hilbert space, and thus it grows exponentially as a function of $N_{B}$. Therefore, for $N_{B} \geqslant 4$, a non-Hermitian Lanczos treatment must be used. The solution of the noninteracting Lindblad problem is nonstandard (see, e.g., Ref. [125]), and a method particularly suited for the present approach is discussed in Sec. II D 1.

\section{Green's functions of the auxiliary Lindblad problem}

In this section, we present expressions for the Green's functions of the auxiliary system. Specifically, we will derive an analytic expression for the noninteracting Green's functions in Sec. IID 1, and illustrate the numerical procedure to determine the interacting ones in Sec. II D 2. The derivations make largely use of the formalism of [126] (see also [127]). For an alternative appealing approach to the noninteracting case, see also Ref. [125]. All Green's functions discussed in Sec. II D are the ones of the auxiliary system, which are different from the physical ones for $N_{B}<\infty$.

The dynamics of the auxiliary open quantum system described by the superoperator $\hat{\mathcal{L}}$ [Eq. (10)] can be recast in an elegant way as a standard operator problem in an augmented fermion Fock space with twice as many sites [125-128]. Specifically, one introduces "tilde" operators $\tilde{d}_{\mu} / \tilde{d}_{\mu}^{\dagger}$ together with the original ones $d_{\mu} / d_{\mu}^{\dagger}$ [129]. Introducing the so-called left vacuum

$$
|I\rangle=\sum_{S}(-i)^{N_{S}}|S\rangle \otimes|\tilde{S}\rangle,
$$

where $|S\rangle$ are many-body states of the original Fock space, $|\tilde{S}\rangle$ the corresponding ones of the tilde space [126], and $N_{S}$ the number of particles in $S$. The nonequilibrium density operator can be written as a state vector in this augmented space

$$
|\rho(t)\rangle \equiv \hat{\rho}(t)|I\rangle
$$

The Lindblad equation is rewritten in a Schrödinger-type fashion $[123,126]$

$$
\frac{d}{d t}|\rho(t)\rangle=\hat{\mathcal{L}}|\rho(t)\rangle
$$

where now $\hat{\mathcal{L}}$ is an ordinary operator in the augmented space. $\hat{\mathcal{L}}=\hat{\mathcal{L}}_{0}+\hat{\mathcal{L}}_{I}$ is conveniently represented in terms of the operators of the augmented space in a vector notation [129]:

$$
\boldsymbol{d}^{\dagger}=\left(d_{0}^{\dagger}, \ldots, d_{N_{B}}^{\dagger}, \tilde{d}_{0}, \ldots \tilde{d}_{N_{B}}\right)
$$

Its noninteracting part $\mathcal{L}_{0}$ reads in the augmented space $[123,126]$ as

$$
i \hat{\mathcal{L}}_{0}=\sum_{\sigma}\left(\boldsymbol{d}^{\dagger} \boldsymbol{h} \boldsymbol{d}-\operatorname{Tr}(\boldsymbol{E}+i \boldsymbol{\Lambda})\right),
$$

where Tr denotes the matrix trace and the matrix $\boldsymbol{h}$ is given by

$$
\boldsymbol{h}=\left(\begin{array}{cc}
\boldsymbol{E}+i \boldsymbol{\Omega} & 2 \boldsymbol{\Gamma}^{(2)} \\
-2 \boldsymbol{\Gamma}^{(1)} & \boldsymbol{E}-i \boldsymbol{\Omega}
\end{array}\right),
$$

with

$$
\boldsymbol{\Lambda}=\left(\boldsymbol{\Gamma}^{(2)}+\boldsymbol{\Gamma}^{(1)}\right), \quad \boldsymbol{\Omega}=\left(\boldsymbol{\Gamma}^{(2)}-\boldsymbol{\Gamma}^{(1)}\right) .
$$

Its interacting part has the form [126]

$$
i \mathcal{L}_{I}=U d_{f \uparrow}^{\dagger} d_{f \uparrow} d_{f \downarrow}^{\dagger} d_{f \downarrow}-U \tilde{d}_{f \uparrow}^{\dagger} \tilde{d}_{f \uparrow} \tilde{d}_{f \downarrow}^{\dagger} \tilde{d}_{f \downarrow} .
$$

In this auxiliary open system, dynamic two-time correlation functions for two operators $\hat{A}$ and $\hat{B}$ of the system can be expressed as

$$
\begin{aligned}
i G_{B A}\left(t_{2}, t_{1}\right) & \equiv\left\langle\hat{B}_{\mathcal{U}}\left(t_{2}\right) \hat{A}_{\mathcal{U}}\left(t_{1}\right)\right\rangle \\
& =\operatorname{tr}_{\mathcal{U}}\left(\hat{B}_{\mathcal{U}}\left(t_{2}\right) \hat{A}_{\mathcal{U}}\left(t_{1}\right) \hat{\rho}_{\mathcal{U}}\right)=\operatorname{tr}\left(\hat{B} \hat{A}_{t_{1}, t_{2}-t_{1}}\right),
\end{aligned}
$$

where $\hat{\rho}_{\mathcal{U}}$ is the density operator of the "universe" $\mathcal{U}$ composed of the system and Markovian environment, tr is the trace over the system degrees of freedom, $\operatorname{tr}_{E}$ the one over the environment, $\operatorname{tr} \mathcal{U}=\operatorname{tr} \otimes \operatorname{tr}_{E}$ the one over the universe, $\hat{O}_{\mathcal{U}}(\ldots)$ denotes the unitary time evolution of an operator $\hat{O}$ according to the Hamiltonian of the universe $\hat{\mathcal{H}}_{\mathcal{U}}$. Here [107],

$$
\hat{A}_{t_{1}, t} \equiv \operatorname{tr}_{E}\left(e^{-i \hat{\mathcal{H}}_{\mathcal{U}} t} \hat{A} \hat{\rho}_{\mathcal{U}}\left(t_{1}\right) e^{+i \hat{\mathcal{H}}_{\mathcal{U}} t}\right) .
$$

Notice that the time evolution of $\hat{\rho}_{\mathcal{U}}(t)$, as well as the one in Eq. (19), are opposite with respect to the Heisenberg time evolution of operators. This is the convention for density operators. For $t=t_{2}-t_{1}>0$ one can use the quantum regression theorem [107] which holds under the same assumptions as for Eq. (9). It states that

$$
\frac{d}{d t} \hat{A}_{t_{1}, t}=\hat{\hat{\mathcal{L}}} \hat{A}_{t_{1}, t}
$$

In the augmented space, in the same way as for (14) and (15), one can associate the operator (19) with the state vector $\left|A_{t_{1}, t}\right\rangle=\hat{A}_{t_{1}, t}|I\rangle$. For this vector, (20) translates into

$$
\frac{d}{d t}\left|A_{t_{1}, t}\right\rangle=\hat{\mathcal{L}}\left|A_{t_{1}, t}\right\rangle \text {. }
$$

Considering its initial value (time $t=0$ )

$$
\left|A_{t_{1}, 0}\right\rangle=\hat{A}\left|\rho\left(t_{1}\right)\right\rangle,
$$

the solution of $(21)$ reads as

$$
\left|A_{t_{1}, t}\right\rangle=e^{\hat{\mathcal{L}} t} \hat{A}\left|\rho\left(t_{1}\right)\right\rangle .
$$

Therefore, we have for the correlation function (18) for $t_{2}>t_{1}$, which we denote as $G_{B A}^{+}\left(t_{2}, t_{1}\right)$ :

$i G_{B A}^{+}\left(t_{2}, t_{1}\right)=\left\langle I\left|\hat{B} e^{\hat{\mathcal{L}}\left(t_{2}-t_{1}\right)} \hat{A}\right| \rho\left(t_{1}\right)\right\rangle=\left\langle I\left|\hat{B}\left(t_{2}-t_{1}\right) \hat{A}\right| \rho\left(t_{1}\right)\right\rangle$,

where

$$
\hat{B}(t):=e^{-\hat{\mathcal{L}} t} \hat{B} e^{\hat{\mathcal{L}} t}
$$

is the non-Hermitian time evolution of the operator $\hat{B}$, and we have exploited the relation [126] $\langle I| \hat{\mathcal{L}}=0$. For the steadystate correlation function, which depends on $t=t_{2}-t_{1}$, we have

$$
i G_{B A}^{+}(t)=\left\langle I|\hat{B}(t) \hat{A}| \rho_{\infty}\right\rangle,
$$


where $\hat{\rho}_{\infty}$ is the steady-state density operator. Since the quantum regression theorem only propagates forward in time, for $t<0$ one has to take the complex conjugate of Eq. (18), which gives for the $t<0$ steady-state correlation function denoted as $G_{B A}^{-}$

$$
i G_{B A}^{-}(t)=-i G_{A^{\dagger} B^{\dagger}}^{+}(-t)^{*}=\left\langle I\left|\hat{A}^{\dagger}(-t) \hat{B}^{\dagger}\right| \rho_{\infty}\right\rangle^{*} .
$$

Using (24), the steady-state greater Green's function for times $t>0$ reads as [130]

$$
\begin{aligned}
G_{\mu \nu}^{>+}(t) & \equiv-i \theta(t)\left\langle d_{\mu}\left(t+t_{1}\right) d_{\nu}^{\dagger}\left(t_{1}\right)\right\rangle_{t 1 \rightarrow \infty} \\
& =-i \theta(t)\left\langle I\left|d_{\mu}(t) d_{\nu}^{\dagger}\right| \rho_{\infty}\right\rangle .
\end{aligned}
$$

We can use (24) also for the lesser Green's function, however, for [130] $t<0$,

$$
\begin{aligned}
G_{\mu \nu}^{<+}(t) & \equiv i \theta(-t)\left\langle d_{\nu}^{\dagger}\left(t_{1}\right) d_{\mu}\left(t+t_{1}\right)\right\rangle_{t 1 \rightarrow \infty} \\
& =i \theta(-t)\left\langle I\left|d_{\nu}^{\dagger}(-t) d_{\mu}\right| \rho_{\infty}\right\rangle .
\end{aligned}
$$

For the opposite sign of $t$, we can use (25), so that for both Green's functions one has [123,130]

$$
\boldsymbol{G}^{\gtrless-}(t)=-\boldsymbol{G}^{\stackrel{2}{<}}(-t)^{\dagger} .
$$

For the Fourier-transformed Green's function, defined, with abuse of notation as

$$
\boldsymbol{G}^{\vec{z} \pm}(\omega)=\int d t e^{i \omega t} \boldsymbol{G}^{\dot{2} \pm}(t),
$$

relation (27) translates into

$$
\boldsymbol{G}^{2-}(\omega)=-\boldsymbol{G}^{2+}(\omega)^{\dagger} .
$$

We need the retarded and the Keldysh Green's functions

$$
\begin{aligned}
& \boldsymbol{G}^{R}=\boldsymbol{G}^{>+}-\boldsymbol{G}^{<-}=\boldsymbol{G}^{>+}+\boldsymbol{G}^{<+\dagger}, \\
& \boldsymbol{G}^{K}=\boldsymbol{G}^{>+}+\boldsymbol{G}^{<-}+\boldsymbol{G}^{>-}+\boldsymbol{G}^{<+}=\boldsymbol{G}^{>+}+\boldsymbol{G}^{<+}-\text {H.c. },
\end{aligned}
$$

whereby both relations hold for the time-dependent as well as for the Fourier-transformed ones.

\section{Noninteracting case}

To solve the noninteracting Lindblad problem described by (16), one first diagonalizes the non-Hermitian matrix [126] $\boldsymbol{h}$ in Eq. (17):

$$
\boldsymbol{\varepsilon}=\boldsymbol{V}^{-1} \boldsymbol{h} \boldsymbol{V},
$$

where $\boldsymbol{\varepsilon}$ is a diagonal matrix of eigenvalues $\varepsilon_{\mu}$. The noninteracting Lindbladian (16) can then be written as

$$
i \hat{\mathcal{L}}_{0}=\bar{\xi} \varepsilon \xi+\eta
$$

in terms of the normal modes

$$
\xi=\boldsymbol{V}^{-1} \boldsymbol{d}, \quad \bar{\xi}=\boldsymbol{d}^{\dagger} \boldsymbol{V}
$$

and a constant $\eta$. The normal modes still obey canonical anticommutation rules

$$
\left\{\xi_{\mu}, \bar{\xi}_{v}\right\}=\delta_{\mu \nu},
$$

but are not mutually Hermitian conjugate.

The steady state $\left|\rho_{\infty}\right\rangle$ obeys the equation

$$
\hat{\mathcal{L}}\left|\rho_{\infty}\right\rangle=0 .
$$

Let us now consider the time evolution (22) of a state initially consisting of the normal mode operators applied to the steadystate density matrix

$$
e^{\hat{\mathcal{L}}_{0} t} \xi_{\mu}\left|\rho_{\infty}\right\rangle=e^{\hat{\mathcal{L}}_{0} t} \xi_{\mu} e^{-\hat{\mathcal{L}}_{0} t}\left|\rho_{\infty}\right\rangle=e^{i \varepsilon_{\mu} t} \xi_{\mu}\left|\rho_{\infty}\right\rangle .
$$

If $\operatorname{Im}\left(\varepsilon_{\mu}\right)<0$, this term diverges exponentially in the longtime limit, which would be in contradiction to the fact that $\left|\rho_{\infty}\right\rangle$ is a steady state, unless the state created by $\xi_{\mu}$ is zero. Therefore, we must have

$$
\xi_{\mu}\left|\rho_{\infty}\right\rangle=0 \quad \text { for } \operatorname{Im}\left(\varepsilon_{\mu}\right)<0 .
$$

Similarly, we must have

$$
\bar{\xi}_{\mu}\left|\rho_{\infty}\right\rangle=0 \quad \text { for } \operatorname{Im}\left(\varepsilon_{\mu}\right)>0 .
$$

These equations, thus, define the steady state as a kind of "Fermi sea." In addition, by requiring that expectation values of the form

$$
\left\langle I\left|\xi_{\mu}(t) \bar{\xi}_{\nu}\right| \rho\right\rangle
$$

do not diverge for large $t$, we obtain that

$$
\begin{array}{ll}
\langle I| \xi_{\mu}=0 & \text { for } \operatorname{Im}\left(\varepsilon_{\mu}\right)>0, \\
\langle I| \bar{\xi}_{\mu}=0 & \text { for } \operatorname{Im}\left(\varepsilon_{\mu}\right)<0 .
\end{array}
$$

From (34d) it follows that an expectation value of the form $\left\langle I\left|\xi_{\mu} \xi_{v}\right| \rho_{\infty}\right\rangle$ vanishes for the case $\operatorname{Im}\left(\varepsilon_{\mu}\right)<0$. For $\operatorname{Im}\left(\varepsilon_{\mu}\right)>$ 0 we make use of the anticommutation rules (33) together with (34b) and the fact that [126] $\left\langle I \mid \rho_{\infty}\right\rangle=\operatorname{tr} \rho_{\infty}=1$ and arrive at

$$
\left\langle I\left|\bar{\xi}_{\mu} \xi_{v}\right| \rho_{\infty}\right\rangle=D_{\mu \nu},
$$

where the matrix

$$
D_{\mu \nu}=\delta_{\mu \nu} \theta\left[\operatorname{Im}\left(\varepsilon_{\mu}\right)\right]
$$

Similarly,

$$
\left\langle I\left|\xi_{\mu} \bar{\xi}_{v}\right| \rho_{\infty}\right\rangle=\bar{D}_{\mu \nu} \equiv \delta_{\mu \nu}-D_{\mu \nu}
$$

The expression for the steady-state correlation functions of the eigenmodes $\boldsymbol{\xi}$ of $\hat{\mathcal{L}}_{0}$ can be now evaluated by considering that, due to the anticommutation rules, the Heisenberg time evolution (23) gives

$$
\xi_{\mu}(t)=e^{-i \varepsilon_{\mu} t} \xi_{\mu}, \quad \bar{\xi}_{\mu}(t)=e^{i \varepsilon_{\mu} t} \bar{\xi}_{\mu} .
$$

Thus,

$$
\left\langle I\left|\xi_{\mu}(t) \bar{\xi}_{v}\right| \rho_{\infty}\right\rangle=e^{-i \varepsilon_{\mu} t}\left\langle I\left|\xi_{\mu} \bar{\xi}_{v}\right| \rho_{\infty}\right\rangle=e^{-i \varepsilon_{\mu} t} D_{\mu \nu} .
$$

In this way, the greater Green's function for $t>0$ becomes

$$
\begin{aligned}
i G_{0 \mu \nu}^{>+}(t) & =\left\langle I\left|d_{\mu}(t) d_{\nu}^{\dagger}\right| \rho_{\infty}\right\rangle \\
& =\sum_{\varsigma} V_{\mu \varsigma} e^{-i \varepsilon_{\varsigma} t} \bar{D}_{\varsigma \varsigma}\left(V^{-1}\right)_{\varsigma \nu} \\
& =\left(\boldsymbol{V} e^{-i \varepsilon t} \overline{\boldsymbol{D}} \boldsymbol{V}^{-1}\right)_{\mu \nu},
\end{aligned}
$$

where we have used (32). The Green's functions are defined with operators $d_{\mu} / d_{\mu}^{\dagger}$ in the original Fock space, so that it is sufficient to know the first $N_{B}+1$ rows (columns) of $\boldsymbol{V}\left(\boldsymbol{V}^{-1}\right)$. For this purpose we introduce

$$
\boldsymbol{U}=\boldsymbol{T} \boldsymbol{V}, \quad \boldsymbol{U}^{(-1)}=\boldsymbol{V}^{-1} \boldsymbol{T}^{\dagger},
$$


whereby $\boldsymbol{T}$ is a $\left(N_{B}+1\right) \times\left(2 N_{B}+2\right)$ matrix, which in block form reads as $\boldsymbol{T}=\left(\begin{array}{ll}1 & 0\end{array}\right)$. Notice that $\boldsymbol{U}^{-1} \neq \boldsymbol{U}^{(-1)}$. With this, the Fourier transform (28) of (35) is given by [131]

$$
\boldsymbol{G}_{0}^{>+}(\omega)=\left(\boldsymbol{U} \frac{\overline{\boldsymbol{D}}}{\omega-\boldsymbol{\varepsilon}} \boldsymbol{U}^{(-1)}\right),
$$

and $G_{0 \mu \nu}^{>-}(\omega)$ is obtained with the help of (29). Similarly, the lesser Green's function for $t<0$

$$
\begin{aligned}
i G_{0 \mu \nu}^{<+}(t) & =-\left\langle I\left|d_{\nu}^{\dagger} d_{\mu}(t)\right| \rho_{\infty}\right\rangle \\
& =-\sum_{\varsigma} V_{\mu \varsigma} e^{-i \varepsilon_{\varsigma} t} D_{\varsigma \varsigma}\left(V^{-1}\right)_{\varsigma \nu} \\
& =-\left(\boldsymbol{U} e^{-i \varepsilon t} \boldsymbol{D} \boldsymbol{U}^{(-1)}\right)_{\mu \nu},
\end{aligned}
$$

with the Fourier transform

$$
\boldsymbol{G}_{0}^{<+}(\omega)=\left(\boldsymbol{U} \frac{\boldsymbol{D}}{\omega-\boldsymbol{\varepsilon}} \boldsymbol{U}^{(-1)}\right)
$$

and $\boldsymbol{G}_{0}^{<-}(\omega)$ is obtained from (29). Using (30) together with (36) and (37), we get

$$
\boldsymbol{G}_{0}^{R}(\omega)=\boldsymbol{U} \frac{\overline{\boldsymbol{D}}}{\omega-\boldsymbol{\varepsilon}} \boldsymbol{U}^{(-1)}+\left(\boldsymbol{U} \frac{\boldsymbol{D}}{\omega-\boldsymbol{\varepsilon}} \boldsymbol{U}^{(-1)}\right)^{\dagger},
$$

and for the Keldysh Green's function using also (29)

$$
\begin{aligned}
\boldsymbol{G}_{0}^{K}(\omega) & =\boldsymbol{U}\left(\frac{\overline{\boldsymbol{D}}}{\omega-\boldsymbol{\varepsilon}}+\frac{\boldsymbol{D}}{\omega-\boldsymbol{\varepsilon}}\right) \boldsymbol{U}^{(-1)}-\text { H.c. } \\
& =\boldsymbol{U}\left(\frac{1}{\omega-\boldsymbol{\varepsilon}}\right) \boldsymbol{U}^{(-1)}-\text { H.c. }
\end{aligned}
$$

In principle, one could just carry out the diagonalization (31) and then evaluate (38) and (39) numerically, which is a rather lightweight task. However, it is possible to obtain a (partially) analytical expression for the Green's functions. Indeed, a lengthy but straightforward calculation yields for the retarded one

$$
\boldsymbol{G}_{0}^{R}(\omega)=(\omega-\boldsymbol{E}+i \boldsymbol{\Lambda})^{-1} .
$$

Similarly, for the Keldysh component of the inverse Green's function, we obtain

$$
\left(\underline{\boldsymbol{G}}_{\mathbf{0}}^{-\mathbf{1}}\right)^{K} \equiv-\boldsymbol{G}_{0}^{R-1} \boldsymbol{G}_{0}^{K} \boldsymbol{G}_{0}^{A-1}=-2 i \boldsymbol{\Omega} .
$$

To sum up, (40) and (41) are the main results of this section. To evaluate $\underline{\Delta}_{\text {aux }}(\omega)$, one then uses (6), whereby one should consider that the matrix $\underline{G}_{0}$ in Keldysh space is just the local one, i.e., in terms of the components local at the impurity $G_{0 f f}^{R}$ and $G_{0 f f}^{K}$ :

$$
\underline{G}_{0} \equiv\left(\begin{array}{cc}
G_{0 f f}^{R} & G_{0 f f}^{K} \\
0 & G_{0 f f}^{A}
\end{array}\right) .
$$

In turn, $G_{0 f f}^{K}$, the $f f$ component of $\boldsymbol{G}_{0}^{K}$, has to be obtained from (41) by the well-known expression [116] $\boldsymbol{G}_{0}^{K}=$ $-\boldsymbol{G}_{0}^{R}\left(\underline{G}_{\mathbf{0}}^{-1}\right)^{K} \boldsymbol{G}_{0}^{A}$.

\section{Interacting case}

The next step consists in solving the interacting auxiliary Lindblad problem described by (10a) in order to determine the
Green's function and the self-energy at the impurity site. This is done by Lanczos exact diagonalization within the many-body augmented Fock space.

First, the steady state $\left|\rho_{\infty}\right\rangle$ has to be determined as the rightsided eigenstate of the Lindblad operator $\hat{\mathcal{L}}$ with eigenvalue $l_{0}=0$. For convenience, we introduce

$$
\hat{L}=i \hat{\mathcal{L}},
$$

which is a kind of non-Hermitian Hamiltonian with complex eigenvalues $\ell$. The dimension of the Hilbert space can be reduced by exploiting symmetries similar to the equilibrium case. The conservation of the particle number per spin $\hat{N}_{\sigma}$ is replaced here by the conservation of $\hat{N}_{\sigma}-\hat{\tilde{N}}_{\sigma}$ [51]. The steady state lies in the sector $N_{\sigma}-\tilde{N}_{\sigma}=0$.

Starting from Eq. (26), the steady-state greater Green's function of the impurity reads as in a non-Hermitian Lehmann representation, for $t>0$,

$$
G_{\mu \nu}^{>+}(t)=-i \sum_{n} e^{-i \ell_{n}^{(+1)} t}\left\langle I\left|d_{\mu}\right| R_{n}^{(+1)}\right\rangle\left\langle L_{n}^{(+1)}\left|d_{\nu}^{\dagger}\right| \rho_{\infty}\right\rangle,
$$

where the identity $\sum_{n}\left|R_{n}^{(+1)}\right\rangle\left\langle L_{n}^{(+1)}\right|$ in the sector $N_{\sigma}-\tilde{N}_{\sigma}=$ +1 has been inserted, in terms of right $\left(\left|R_{n}^{(+1)}\right\rangle\right)$ and left $\left(\left\langle L_{n}^{(+1)}\right|\right)$ eigenstates of $\hat{L}$ with eigenvalues $\ell_{n}^{(+1)}$, and $|I\rangle$ is the left vacuum (13). Its Fourier transform reads as

$$
\begin{aligned}
G_{\mu \nu}^{>}(\omega)= & \sum_{n} \frac{1}{\omega-\ell_{n}^{(+1)}}\left\langle I\left|d_{\mu}\right| R_{n}^{(+1)}\right\rangle\left\langle L_{n}^{(+1)}\left|d_{\nu}^{\dagger}\right| \rho_{\infty}\right\rangle \\
& -\sum_{n} \frac{1}{\omega-\ell_{n}^{(+1) *}}\left(\left\langle I\left|d_{\nu}\right| R_{n}^{(+1)}\right\rangle\left\langle L_{n}^{(+1)}\left|d_{\mu}^{\dagger}\right| \rho_{\infty}\right\rangle\right)^{*} .
\end{aligned}
$$

The analogous expression for the lesser Green's function $G_{\mu \nu}^{<}(\omega)$ is obtained by inserting a complete set of eigenstates in the $N_{\sigma}-\tilde{N}_{\sigma}=-1$ sector and exchanging the elementary operators accordingly. $G_{\mu \nu}^{K}(\omega)$ and $G_{\mu \nu}^{R}(\omega)$ are obtained using Eq. (30) [see also (29)].

For a small number of bath sites $N_{B} \leqslant 3$, the dimension of the augmented Fock space is still moderate, and eigenvalues and eigenvectors can be determined by full diagonalization. For $N_{B} \geqslant 4$, a non-Hermitian Lanczos procedure has to be carried out. Especially extracting the steady state is not an easy task since it lies in the center of the spectrum. Details of our numerical procedure are given in Appendix A.

Once the interacting and noninteracting Green's functions of the auxiliary system at the impurity site $\underline{G}(\omega)$ and $\underline{G}_{0}(\omega)$, respectively, are determined, the corresponding self-energy is obtained via Dyson's equation in Keldysh space [Eq. (5)]. The individual components are explicitly [51]

$$
\begin{aligned}
& \Sigma^{R}(\omega)=1 / G_{0}^{R}(\omega)-1 / G^{R}(\omega), \\
& \Sigma^{K}(\omega)=-G_{0}^{K}(\omega) /\left|G_{0}^{R}(\omega)\right|^{2}+G^{K}(\omega) /\left|G^{R}(\omega)\right|^{2} .
\end{aligned}
$$

As discussed in Sec. II B, this is used in the Dyson equation (5) for the physical Green's function.

\section{RESULTS}

In this section, results for the steady-state properties of a symmetric, correlated Anderson impurity coupled to two 

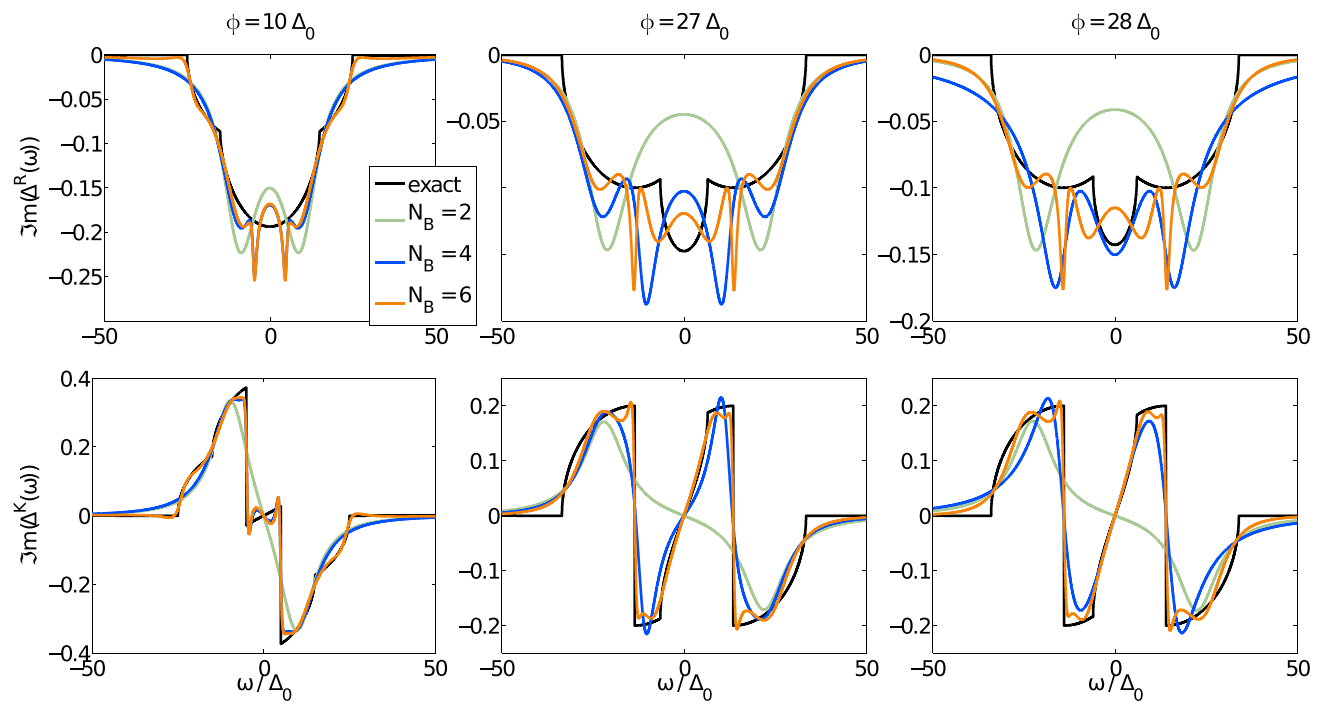

FIG. 2. (Color online) Comparison of $\operatorname{Im}\left[\Delta^{\alpha}(\omega)\right]$ from (7) (black) with $\operatorname{Im}\left[\Delta_{\text {aux }}^{\alpha}(\omega)\right]$ at the absolute minimum of the cost function (12) for auxiliary system sizes $N_{B}=2,4$, and 6 bath sites (green, blue, and orange, respectively), and $\alpha=R$ (top) and $K$ (bottom). Results are shown for tight-binding leads (2) and (8) with $t=10 \Delta_{0}$, and three different bias voltages $\phi \in\{10,27,28\} \Delta_{0}$ from left to right.

metallic leads under bias voltage are provided. We assess the validity of the proposed method by discussing the fit of the hybridization function and outline how uncertainties are estimated. Results for the current voltage characteristics and the nonequilibrium spectral function are presented and compared with data from time-evolving block decimation (TEBD) [77] and SNRG [54] calculations, respectively. The effect of a linear correction of the calculated Green's functions is illustrated.

\section{A. Hybridization functions}

The optimal representation of the exact bath $\underline{\Delta}(\omega)$ by the auxiliary one $\Delta_{\text {aux }}(\omega)$ is obtained by minimizing the cost function (12). In practice, this is done by employing a quasi-Newton line search [132,133]. In particular, we chose an equal weighting of the retarded and the Keldysh component $W^{R}(\omega)=W^{K}(\omega)=\Theta\left(\omega_{c}-|\omega|\right)$. After finding our results to be robust upon different values for the cutoff $\omega_{c}$, as well as upon using different norms $(n=1,2)$ in Eq. (12), we finally choose $\omega_{c}=50 \Delta_{0}$ and consider imaginary parts $\left\{\operatorname{Im}\left[\Delta^{\alpha}(\omega)-\Delta_{\text {aux }}^{\alpha}(\omega)\right]\right\}^{2}$ in the cost function only. This is justified since $\Delta_{\text {aux }}^{K}(\omega)$ is purely imaginary and the real part of $\Delta_{\text {aux }}^{R}(\omega)$ is connected to its imaginary part via the KramersKronig relations [134]. The asymptotic behavior of $\Delta_{\text {aux }}^{R}(\omega)$ is determined by $\Lambda_{f f}$ whereas the one of $\Delta_{\text {aux }}^{K}(\omega)$ by $\Omega_{f f}$. Therefore, the correct asymptotic $\operatorname{limit}_{\lim } \lim _{\omega \rightarrow \infty} \Delta_{\text {aux }}(\omega)=$ $\underline{0}$ is guaranteed by taking $\Gamma_{f f}^{(1)}=\Gamma_{f f}^{(2)}=0$, which results in $\Gamma_{\mu f}^{(\kappa)}=\Gamma_{f \mu}^{(\kappa)}=0$ due to the requirement of semipositive definiteness of $\Gamma_{\mu \nu}^{(\kappa)}$. Particle-hole symmetry allows for a further reduction of the auxiliary system parameters [135].

In this work, we use an even number of auxiliary bath sites $N_{B}=2,4$, and 6 in a linear setup [see Fig. 1(b)] with an equal number to the left and to the right of the impurity (only Fig. 6 displays one calculation for an odd number of bath sites). In Fig. 2, the obtained auxiliary hybridization functions are compared with the exact ones for various bias voltages. We find a quick convergence as a function of $N_{B}$, which degrades for large bias voltage $\phi$. The Fermi steps at the chemical potentials in $\Delta_{\text {aux }}^{K}(\omega)$ can not be properly resolved in the case of $N_{B}=2$. Especially in the case of $\phi=10 \Delta_{0}$ the auxiliary hybridization functions for $N_{B}=6$ as well as for $N_{B}=4$ agree fairly well with the exact one and capture all essential features, in particular the Fermi steps. The auxiliary bath develops spurious oscillations in $\Delta_{\text {aux }}^{R}(\omega)$ at the energies of the Fermi levels of the contacts. Here, the discrepancy with $\Delta^{R}(\omega)$ is considerable in magnitude, but extends over small $\omega$ intervals, thus inducing only small errors in the self-energies.

When following the absolute minimum of the cost function (12) as a function of some external parameter, such as, e.g., the bias voltage $\phi$, spurious discontinuities appear due to the fact that local minima cross each other. This occurs for large bias voltages and large $U$, and/or small $N_{B}$, for which the approach is more challenging. An example for such a situation is shown in Fig. 2 for the case $N_{B}=4$, when comparing the hybridization functions just before and after such a crossing, i.e., for $\phi=27 \Delta_{0}$ and $28 \Delta_{0}$. Even though the changes in the exact hybridization function are only minor, $\underline{\Delta}_{\text {aux }}(\omega)$ displays a considerable difference. The influence of this spurious effect on observable quantities is shown in Fig. 3 (right panel, orange circles) for a different parameter set of $N_{B}=6$ at around $\phi_{c}=33 \Delta_{0}$. The artificial discontinuity in the current is caused by the shift of spectral weight in $\Delta_{\text {aux }}(\omega)$.

To deal with these discontinuities, we adopt a scheme which is suitable for obtaining a continuous dependence of observables on external parameters and, in addition, allows us to estimate their uncertainties (see Fig. 3). We first identify a set of local minima of the cost function (12), obtained by a series of minimum searches starting with random initial values. These local minima are then used to calculate an average and variance of physical quantities, such as the current. We consider the distribution of local minima with a Boltzmann weight associated with an artificial "temperature," whereby the value of the cost function (12) is the associated 


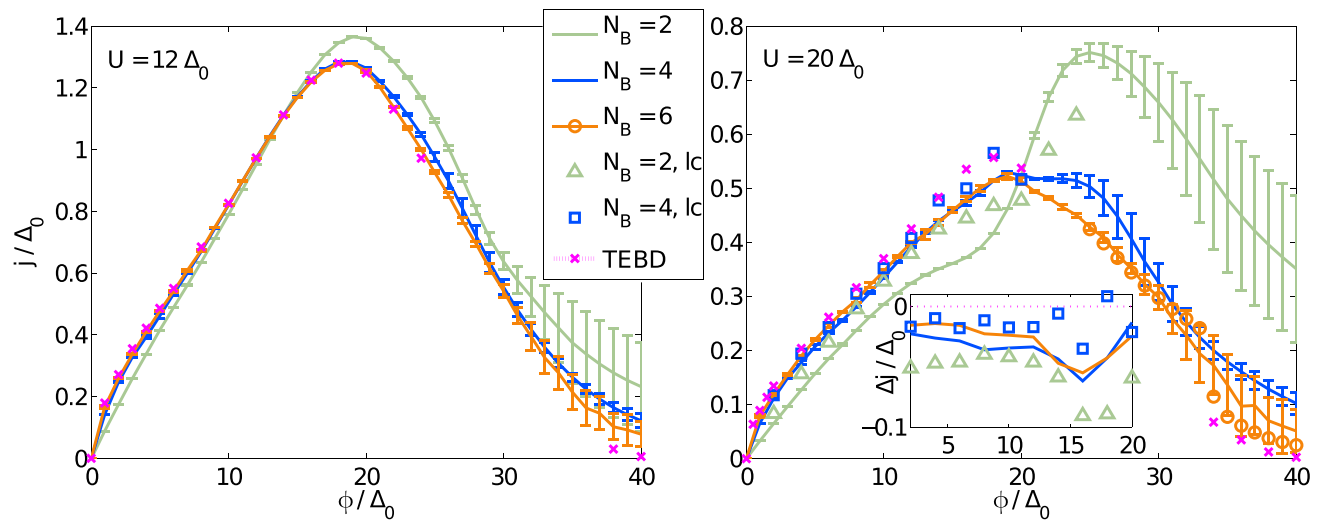

FIG. 3. (Color online) Current $j$ vs voltage $\phi$ for the model (1) with tight-binding leads and onsite interaction $U=12 \Delta_{0}$ (left) and $U=20 \Delta_{0}$ (right). Results for three different auxiliary systems with $N_{B} \in\{2,4,6\}$ are displayed and compared with reference data from TEBD (magenta dotted and $\times$ ) [77]. We plot the averaged mean values connected by lines together with error bars determined according to Sec. III A and Appendix B. The additional data marks for $U=20 \Delta_{0}$ are as follows: The circles for $N_{B}=6$ display $j(\phi)$ when considering the absolute minimum in the fit (12). $N_{B}=2$, lc and $N_{B}=4$, lc present the results of a linear correction of the current values of the absolute minima as described in Appendix C. The inset displays the difference $\Delta j$ of the calculated currents to the TEBD results.

"energy." This artificial temperature for the Boltzmann weight is chosen in such a way that the averaged spectral weight of the hybridization function as a function of $\phi$ is as smooth as possible. Details are outlined in Appendix B. A possible pitfall, however, is that physical discontinuities, i.e., real phase transitions could be overlooked. It is thus compulsory to additionally investigate the results for the absolute minima and for different bath setups carefully. This approach has a certain degree of arbitrariness. However, we point out that it only affects regions with large error bars in Fig. 3, i.e., large $\phi$ and large $U$ for which also other techniques are less accurate.

\section{B. Current-voltage characteristics}

After evaluating the interacting impurity Green's function of the physical system according to (5) with the self-energy evaluated in Sec. IID, we are able to determine the steadystate current. This is done with the help of the Meir-Wingreen expression [116,136,137] in its symmetrized form, where we have already summed over spin

$$
\begin{aligned}
j= & i \int_{-\infty}^{\infty} \frac{d \omega}{2 \pi}\left(\left[\gamma_{L}(\omega)-\gamma_{R}(\omega)\right] G^{<}(\omega)\right. \\
& \left.+\left[p_{\mathrm{F}, L}(\omega) \gamma_{L}(\omega)-p_{\mathrm{F}, R}(\omega) \gamma_{R}(\omega)\right]\left[G^{R}(\omega)-G^{A}(\omega)\right]\right),
\end{aligned}
$$

$\gamma_{\lambda}(\omega)=-2\left|t_{\lambda}^{\prime}\right|^{2} \operatorname{Im}\left[g_{\lambda}^{R}(\omega)\right]$ are the "lead self-energies" and $p_{\mathrm{F}, \lambda}(\omega)=p_{\mathrm{F}}\left(\omega-\mu_{\lambda}\right)$ denotes the Fermi distribution of lead $\lambda$ with chemical potential $\mu_{\lambda}$.

To quantify the accuracy of the method, we compare the results for the current-voltage characteristics with quasiexact reference data from TEBD [77]. We find very good agreement for interaction strength $U<12 \Delta_{0}$. Since in this paper we want to benchmark the approach in "difficult" parameter regimes, in the following, we will discuss $U \gtrsim 12 \Delta_{0}$ only. In Fig. 3 we display data for $U=12 \Delta_{0}$ and $20 \Delta_{0}$. The data points and error bars shown are obtained by using the averaging scheme as described in Appendix B. For the universal physics at small and medium bias voltages $\phi \lesssim 20 \Delta_{0}$, the current as a function of the auxiliary system size $\left(N_{B} \in\{2,4,6\}\right)$ converges rapidly to the expected result. The convergence is even monotonic in a broad region of the parameter space. The zero-bias response is linear for all $N_{B}$ and approaches the results expected from the Friedel sum rule [29] $j\left(\phi=0^{+}\right)=2 \frac{e^{2}}{h} \phi$ quickly for increasing $N_{B}$. For $U=12 \Delta_{0}$ already the $N_{B} \gtrsim 4$ results yield a good reproduction of the current in this bias regime. For $U=20 \Delta_{0}$ and $\phi \gtrsim 20 \Delta_{0}$, a larger difference between the $N_{B}=4$ and 6 results is observed. Notice that also other available methods do not yield a satisfactory result in this parameter regime. In the lead-dependent high-bias regime, the fit becomes more challenging and large variances appear in the calculated quantities. This indicates the presence of many competing local minima with similar values for the cost function whose value tends to increase with increasing $\phi$. For $\phi \geqslant 40 \Delta_{0}$, the densities of states of the left and the right contacts do not overlap anymore and the current has to vanish. This limit can not be exactly reproduced by the proposed approach due to spurious long-range Lorentzian tails present in the auxiliary Markovian environment. Nevertheless, $j\left(\phi=40 \Delta_{0}\right)$ approaches zero as one increases the number of bath sites. This holds true for quantities obtained at the absolute minimum of the cost function as well as for averaged ones.

To extrapolate our results to larger $N_{B}$, a scheme for linear corrections is discussed in Appendix C. Data for $N_{B}=2, \mathrm{lc}$ and 4,lc, whereby "lc" denotes "linear correction," is shown in Fig. 3. For large $U=20 \Delta_{0}$ and small- to medium-bias voltages $\phi \lesssim 20 \Delta_{0}$, a solid improvement towards the TEBD reference values is observed (see inset Fig. 3). Correction ratios $r$ (see Appendix $\mathrm{C}$ ) close to one indicate a good applicability of the linear correction scheme. We find on average $r \approx 0.75$ for $\phi \lesssim 20 \Delta_{0}\left(N_{B}=2\right.$,lc and 4,lc). In the high-bias regime, however, the linear correction can not be applied with large magnitude and $r$ drops below 0.5 for $N_{B}=2$,lc. Nevertheless, the calculation of the effective, auxiliary hybridization function $\underline{\Delta}_{\mathrm{aux}, r}(\omega)$ as described in Appendix C successfully avoids an "overcorrection" of the current values and automatically allows one to estimate the reliability of the results. 

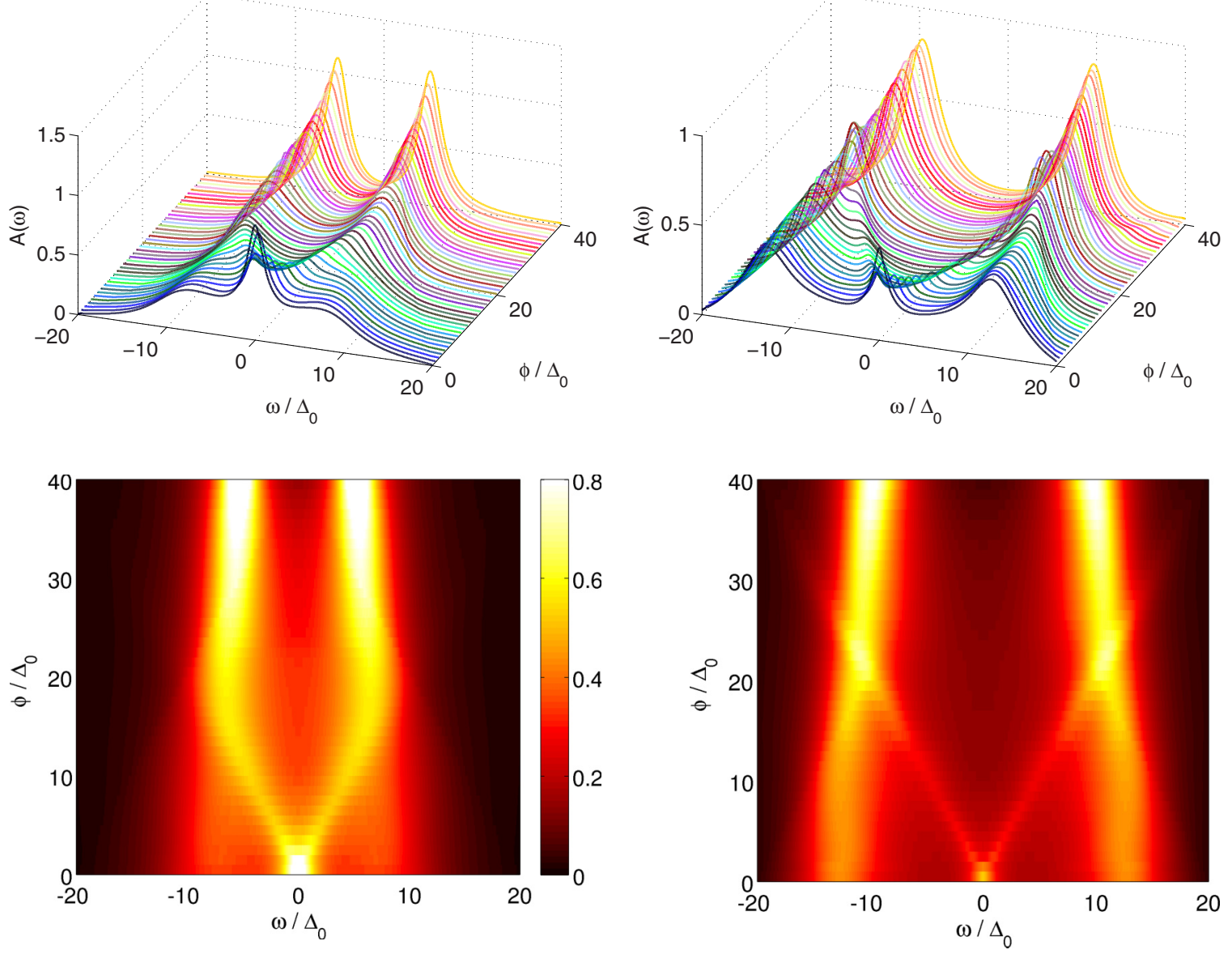

FIG. 4. (Color online) Single-particle spectral function at the impurity evaluated for $N_{B}=6$, different bias voltages $\phi$, and $U=12 \Delta_{0}$ (left) and $U=20 \Delta_{0}$ (right). Data are obtained according to Sec. III A and Appendix B. Other parameters are as in Fig. 3.

Judging from the larger uncertainty from the averaging procedure and the strong effects of the linear corrections, we conclude that the high-bias regime is more sensitive to the details of the fitted, auxiliary hybridization function. The universal low- and medium-bias regimes are, however, very well reproduced even with a small number of auxiliary bath sites.

\section{Nonequilibrium spectral function}

The bias-dependent single-particle spectral function is evaluated from the physical steady-state Green's function of the impurity $A(\omega)=-\frac{1}{\pi} \operatorname{Im}\left[G^{R}(\omega)\right]$. Results obtained using $N_{B}=6$ for $U=12 \Delta_{0}$ and $20 \Delta_{0}$ are presented for the whole bias range of interest in Fig. 4. Data for $N_{B}=4$ are similar, but here the Kondo physics can not be reproduced as accurately as in the case of $N_{B}=6$. Our approach does preserve the local charge density $\left\langle n_{f}\right\rangle=\sum_{\sigma} \frac{1}{2}+\frac{1}{2} \int_{-\infty}^{\infty} \frac{d \omega}{2 \pi} \operatorname{Im}\left[G^{K}(\omega)\right]=1$ and magnetization $\left\langle m_{f}\right\rangle=0$ as well as the spectral sum rule [138].

The presented method reproduces qualitatively correctly also the equilibrium physics at $\phi=0$ since $A(\omega)$ displays a Kondo resonance at $\omega=0$ and two Hubbard satellites at the approximate positions $\omega \approx \pm U / 2$. This renders the application to equilibrium DMFT problems an interesting perspective. The width and magnitude of the Kondo resonance are discussed in comparison with (S)NRG data in Sec. III C 1.
Upon increasing the bias voltage, the Kondo resonance splits up and two excitations are observed at the energies of the Fermi levels of the leads [78,139,140]. For $U=12 \Delta_{0}$, the splitted resonances merge into the Hubbard bands at approximately $\phi \approx 15 \Delta_{0}$ and can not be clearly identified thereafter. In contrast, in the case of $U=20 \Delta_{0}$, the resonances overlap with the Hubbard satellites and can still be observed in the spectrum $A(\omega)$ at higher voltages. Calculations with increasing $U$ in the high-bias regime $\phi \approx 40 \Delta_{0}$ have shown the consistency of this effect and that a minimum value of $U \approx 15 \Delta_{0}$ is needed in order for the resonances at the Fermi energies to be perceptible after having crossed the Hubbard bands.

\section{Comparison with scattering states numerical renormalization group}

We compare the computed spectral functions with results obtained by means of SNRG [53]. For this purpose, we use a flat DOS [Eq. (3)] for the leads, as in Ref. [53]. Focusing on the low-bias regime and $N_{B}=6$, the obtained spectral functions are depicted in Fig. 5. Compared with SNRG, our results do not achieve the same accuracy in the low-energy domain, i.e., in the vicinity of $\omega \approx 0$. However, our data provide a better resolution at higher energies. When inspecting the Kondo peak in the equilibrium case $\phi=0$, our results do not fully fulfill the Friedel sum rule [29,141,142]. Depending on parameters, 


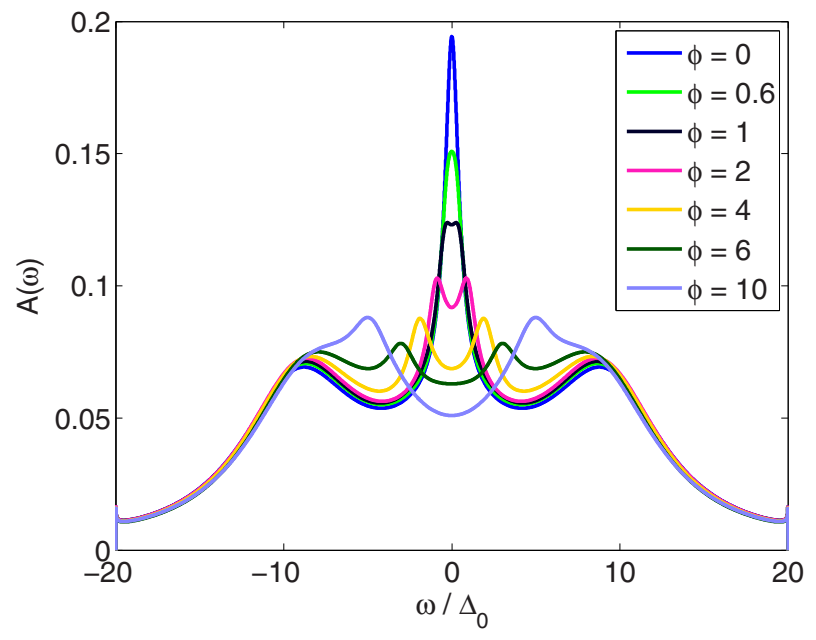

FIG. 5. (Color online) Single-particle spectral function for a constant DOS of the leads (3), with $D_{\mathrm{WB}}=20 \Delta_{0}$ and $U=16 \Delta_{0}$ and different bias voltages $\phi$ (in units of $\Delta_{0}$ ). Results are obtained for $N_{B}=6$ and at the absolute minimum of Eq. (12). For a comparison with SNRG [53] [Fig. 2(a) therein], note that their $\Gamma=2 \Delta_{0}$.

the height of the Kondo resonance is underestimated. This is due to the fact that the imaginary part of the self-energy at $\omega=0$ has a small finite value which is due to the Lorentzian tails of the Markovian environment.

The resolution does not suffice to tell whether a two- or a three-peak structure is present for very low-bias voltages $\phi \lesssim$ $2 \Delta_{0}$. Nevertheless, one can say that the higher-bias regime $\phi>4 \Delta_{0}$ is resolved more accurately and one is able to clearly distinguish the excitations at the Fermi energies of the contacts from the Hubbard satellites. The observed linear splitting is consistent with experiments on nanodevices [139,140]. Within second-order Keldysh PT [58] and QMC results [143], the resonance does not split but is suppressed only. In fourth order and in NCA it splits into two, which are located near the chemical potentials of the two leads [58]. Other methods yield a splitting with features slightly different in details: real-time diagrammatics [144], VCA [78], imaginary potential QMC [90], or scaling methods [145]. Overall, a good qualitative agreement with the SNRG results is achieved which underlines the reliability of the calculated spectral functions.

\section{Linear correction of Green's functions}

Here, we consider the effect of a linear correction of the Green's functions, as outlined in Appendix C. In the left panels (right panels) of Fig. 6, we show data for $N_{B}=2$ $\left(N_{B}=4\right)$ including linear corrections $(r=1)$ for a high interaction strength in the low-bias regime. We benchmark to data obtained using $N_{B}=6$ without corrections.

For $N_{B}=2$ without linear corrections, the spectral function of the auxiliary system does not feature excitations at the Fermi energies of the contacts $\left(\omega= \pm 2 \Delta_{0}\right)$, which are present in the $N_{B}=6$ data. Also, the spectra appear washed out. The linearly corrected result, however, features not only the two resonances at the appropriate energies, but also the shoulders present in the reference data. Again, in the Keldysh Green's
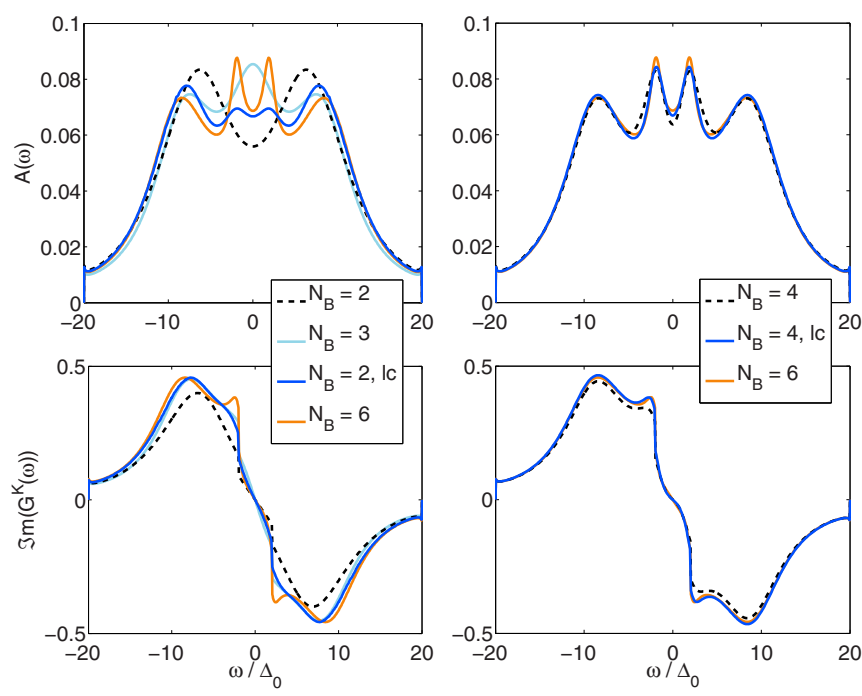

FIG. 6. (Color online) Effects of the linear corrections of the Green's functions according to Apendix $\mathrm{C}$ (solid blue lines). The dashed lines indicate the uncorrected $\underline{G}(\omega)$ with the same $N_{B}$, while solid orange and light blue lines display results for larger $N_{B}$ for comparison. Results are shown for a constant lead DOS [Eq. (3)] with $D_{\mathrm{WB}}=20 \Delta_{0}, U=16 \Delta_{0}$, and $\phi=4 \Delta_{0}$.

function a large correction towards the more accurate $N_{B}=6$ results is observed. To highlight the fact that the improvement of the linear correction is not only due to the inclusion of one additional bath site, also a calculation for an auxiliary system with $N_{B}=3$ is shown. Evidently, the $N_{B}=3$ spectral function exhibits a large weight at low frequencies, but the resolution is rather low and only a single, smeared out peak at $\omega=0 \Delta_{0}$ is observed. It clearly does not account for the splitting of the Kondo resonance.

For $N_{B}=4$, a similar enhancement is found. Clearly, the size of the corrections is much smaller. Especially in the Keldysh component, the Green's function for $N_{B}=6$ and for the corrected $N_{B}=4$ system nearly coincide. In general, the difference between the $N_{B}=6$ and the $N_{B}=4$ calculations (raw and corrected) is quite small, so that the presented spectral functions in Fig. 5 for larger values of $\phi \lesssim 12 \Delta_{0}$ can be assumed to be quite accurate.

Overall, the linear correction enables a vast improvement in the universal low- and medium-bias regimes for all $U$, which becomes especially important for large $U$. For large-bias voltages, when lead band effects become prominent, the linear correction is more challenging (see also Sec. III B).

\section{CONCLUSIONS}

We have presented a numerical approach to study correlated quantum impurity problems out of equilibrium [51]. The auxiliary master equation approach presented here is based on a mapping of the original Hamiltonian to an auxiliary open quantum system consisting of the interacting impurity coupled to bath sites as well as to a Markovian environment. The dynamics of the auxiliary open system is controlled by a Lindblad master equation. Its parameters are determined by a fit to the impurity-environment hybridization function. 
This has many similarities to the procedure used for the exact-diagonalization dynamical mean field theory impurity solver, but has the advantage that one can work directly with real frequencies, which is mandatory for nonequilibrium systems.

We have illustrated how the accuracy of the results can be estimated, and systematically improved by increasing the number of auxiliary bath sites. A scheme to introduce linear corrections has been devised. We presented in detail how the nonequilibrium Green's functions of the correlated open quantum system are obtained by making use of non-Hermitian Lanczos diagonalization in a superoperator space. These techniques make the whole method fast and efficient as well as particularly suited as an impurity solver for steady-state dynamical mean field theory [51].

In this work, we have applied the approach to the singleimpurity Anderson model, which is one of the paradigmatic quantum impurity models. We have analyzed in detail the systematic improvement of the current-voltage characteristics as a function of the number of auxiliary bath sites. Already for four auxiliary bath sites, results show a rather good agreement with quasiexact data from time-evolving block decimation [77] in the low- and medium-bias regimes. In the high-bias regime, the current deviates from the expected result with increasing interaction strength. However, we have shown how to estimate the reliability of the data from the deviation of the hybridization functions and how results can be corrected to linear order in this deviation. The impurity spectral function obtained in our calculation features a linear splitting of the Kondo resonance as a function of bias voltage. Good agreement with data from scattering-state numerical renormalization group [53] was found.

Applications of the presented method to multiorbital correlated impurities or correlated clusters is in principle straightforward, although numerically more demanding. Such systems are themselves of interest as models for transport through molecular or nanoscopic objects and as solvers for nonequilibrium cluster dynamical mean field theory. In this case, a larger number of auxiliary sites might be necessary to obtain a good representation of the various hybridization functions. For this situation, one should use numerically more efficient methods to solve for larger correlated open quantum systems, such as matrix product states and density matrix renormalization group, possibly combined with stochastic wave-function approaches [146-148], sparse polynomial space $[149,150]$, or configuration interaction approaches [151]. A more accurate determination of low energy, and possibly critical properties, might be achieved by a combination with renormalization group iteration schemes, similar to the numerical renormalization group. Work along these lines is in progress.

Although we have presented results for the steady state, where the method is most efficient, also extensions to timedependent phenomena provide an interesting and feasible perspective. While other approaches, such as time-dependent density matrix renormalization group [73] or quantum Monte Carlo [49] are certainly more accurate at short times, the present approach could be used to estimate directly slowly decaying modes by inspecting the behavior of the low-lying spectrum of the Lindblad operator.

\section{ACKNOWLEDGMENTS}

We acknowledge discussions with A. Rosch, W. Hofstetter, S. Diehl, M. Knap, D. Rost, and F. Schwarz. A.D. and M.N. thank the Forschungszentrum Jülich, in particular the autumn school on correlated electrons, for hospitality and support. This work was supported by the Austrian Science Fund (FWF), Grants No. P24081-N16 and No. P26508-N20, as well as SfBViCoM Project No. F04103.

\section{APPENDIX A: NUMERICAL CALCULATION OF THE AUXILIARY INTERACTING GREEN'S FUNCTION}

In this section, we present details of the numerical evaluation of the auxiliary Green's function, as described in Sec. II D 2. We focus on large Hilbert spaces for which a sparse-matrix approach is mandatory. To determine the steady state, which is the right-sided eigenstate of $\hat{L}$ with eigenvalue zero, one can make use of a shift-and-invert Arnoldi procedure [152-155]. The spectrum of $\hat{L}$ [Eq. (42)] has the property that $\operatorname{Im}\left(\ell_{n}\right)<0$ for all eigenvalues $\ell_{n}$ (except the steady state $\left.\ell_{0}=0\right)$. Therefore, given a small shift $s>0$, the eigenvector of $(\hat{L}-i s \hat{1})^{-1}$ with the largest eigenvalue is the steady state. Since $\hat{L}$ is non-Hermitian, the three-term recurrence of the ordinary Lanczos scheme [120] does not apply, and one has to resort to an Arnoldi scheme instead. To construct the corresponding Krylov space, a system of equations $(\hat{L}-i s \hat{11})\left|\tilde{\phi}_{n+1}\right\rangle=\left|\phi_{n}\right\rangle$ has to be solved in each step. For the problem at hand, we found that this can be done most efficiently by combining a stabilized biconjugate gradient method with an incomplete LU decomposition as preconditioner $[156,157]$. Despite using sparse-matrix methods, the memory requirements of this approach are rather high compared to the schemes presented below.

A second possible route to determine the steady state $\left|\rho_{\infty}\right\rangle$ is to perform an explicit time evolution. For unitary time evolutions, a well-established method relies on the Lanczos scheme to construct an approximate time evolution operator [158]. Such an approach can be adapted to the nonunitary case by using a two-sided Lanczos scheme (see below), or also by employing an Arnoldi procedure [155]. Since $\hat{L}$ is non-Hermitian, one can equally well use a simpler backward or forward Euler scheme [133] to discretize the nonunitary time evolution operator. These approaches may not yield a highly accurate time evolution of $|\rho(t)\rangle$, but can nevertheless determine the steady state within a moderate number of steps. As for the shift-and-invert approach above, to solve the implicit update of $\left|\rho\left(t_{n+1}\right)\right\rangle$ at time step $t_{n}$ in the case of the backward Euler, a biconjugate gradient routine has proven to be effective. For the forward time integration, a Runge-Kutta method of second order is sufficient, with the great advantage that only matrix-vector multiplications are needed, which reduces memory requirements. In practice, for the considered cases it was found that for not too large systems $\left(N_{B}<6\right)$, the shift-and-invert Arnoldi procedure is best suited, whereas a forward time integration is advantageous for the case $N_{B}=6$.

Once the steady state is determined, Green's functions can be effectively calculated by employing a two-sided Lanczos scheme [152,159-163]. We therefore express the right- and 
left-sided eigenvectors of $\hat{L}$ in Eq. (43) in a Krylov space basis

$$
\left|R_{n}\right\rangle=\sum_{k} U_{k n}\left|\phi_{R}^{k}\right\rangle, \quad\left\langle L_{n}\right|=\sum_{k}\left(U^{-1}\right)_{n k}\left\langle\phi_{L}^{k}\right| .
$$

Here, we have omitted the $N_{\sigma}-\tilde{N}_{\sigma}$ symmetry sector index for the sake of clarity. The biorthogonal Lanczos vectors

$$
\left\langle\phi_{L}^{k} \mid \phi_{R}^{k^{\prime}}\right\rangle=\delta_{k k^{\prime}}
$$

are determined by the three-term recurrence

$$
\begin{aligned}
\left|\phi_{R}^{n+1}\right\rangle & =\frac{1}{c_{n+1}}\left(\hat{L}\left|\phi_{R}^{n}\right\rangle-e_{n}\left|\phi_{R}^{n}\right\rangle-k_{n}\left|\phi_{R}^{n-1}\right\rangle\right), \\
\left\langle\phi_{L}^{n+1}\right| & =\frac{1}{c_{n+1}^{*}}\left(\left\langle\phi_{L}^{n}\right| \hat{L}-e_{n}\left\langle\phi_{L}^{n}\right|-k_{n}^{*}\left\langle\phi_{L}^{n-1}\right|\right),
\end{aligned}
$$

with

$$
\begin{aligned}
& e_{n}=\left\langle\phi_{L}^{n}|\hat{L}| \phi_{R}^{n}\right\rangle, \\
& k_{n}=\left\langle\phi_{L}^{n-1}|\hat{L}| \phi_{R}^{n}\right\rangle=\left(\left|\phi_{L}^{n}\right| \hat{L}\left|\phi_{R}^{n-1}\right\rangle\right)^{*},
\end{aligned}
$$

and a normalization constant $c_{n}$ such that $\left\langle\phi_{L}^{n} \mid \phi_{R}^{n}\right\rangle=1$. One has a certain degree of freedom in the choice of $c_{n}$ and $k_{n}$ due to the relation $k_{n}^{*}=c_{n}$, which is fulfilled, for example, by $k_{n}=k_{n}^{*}=c_{n}$.

In the Krylov basis, $\hat{L}$ takes on a tridiagonal form $T_{n m}=$ $\left\langle\phi_{L}^{n}|\hat{L}| \phi_{R}^{m}\right\rangle$ with the matrix elements $T_{n n}=e_{n}, T_{n-1 n}=k_{n}$, and $T_{n n-1}=k_{n}^{*}$. When $n+1$ becomes as large as the degree of the minimal polynomial of $\hat{L}$, the eigenvalues and eigenvectors of $T$ represent those of $\hat{L}[152,160]$. If one truncates the Krylov basis, this statement holds still approximately true, especially for the largest eigenvalues in magnitude. Analogous to the Hermitian case [164], an exponential convergence of the eigenspectrum of $T$ towards the one of $\hat{L}$ is observed, which is of particular importance for the calculation of Green's functions. A peculiarity of the two-sided Lanczos scheme is that not every Krylov subspace guarantees that $\operatorname{Im}\left(\ell_{n}\right)<0$ for all eigenvalues $\ell_{n}$ of $T$. In order to obtain the appropriate pole structure for the estimated Green's functions, one has to check $\operatorname{Im}\left(\ell_{n}\right)<0$ together with convergence criteria. In cases in which $\operatorname{Im}\left(\ell_{n}\right)<0$ can not be fulfilled exactly, it has to be ensured at least that the corresponding weights of these eigenvalues are negligible.

For the calculation of the Green's functions needed here it is convenient to choose appropriate initial vectors, which are in the case of the greater Green's function (43)

$$
\left|\phi_{R}^{0}\right\rangle=\frac{1}{c_{0}}\left(d_{f}^{\dagger}\left|\rho_{\infty}\right\rangle\right), \quad\left\langle\phi_{L}^{0}\right|=\frac{1}{c_{0}^{*}}\left(\langle I| d_{f}\right) .
$$

When denoting by $\ell_{n}$ and $U_{k, n}$ the eigenvalues and right-sided eigenvectors of $T$, respectively, Eq. (43) can be cast into the form

$$
\begin{aligned}
G^{>}(\omega)= & \sum_{n, k, k^{\prime}} \frac{U_{k n} U_{n k^{\prime}}^{-1}}{\omega-\ell_{n}}\left\langle I\left|d_{f}\right| \phi_{R}^{k}\right\rangle\left\langle\phi_{L}^{k^{\prime}}\left|d_{f}^{\dagger}\right| \rho_{\infty}\right\rangle \\
& -\sum_{n, k, k^{\prime}} \frac{\left(U_{k n} U_{n k^{\prime}}^{-1}\right)^{*}}{\omega-\ell_{n}^{*}}\left(\left\langle I\left|d_{f}\right| \phi_{R}^{k}\right\rangle\left\langle\phi_{L}^{k^{\prime}}\left|d_{f}^{\dagger}\right| \rho_{\infty}\right\rangle\right)^{*} \\
= & \left|c_{0}\right|^{2} \sum_{n} \frac{U_{0 n} U_{n 0}^{-1}}{\omega-\ell_{n}}-\left|c_{0}\right|^{2} \sum_{n} \frac{\left(U_{0 n} U_{n 0}^{-1}\right)^{*}}{\omega-\ell_{n}^{*}} .
\end{aligned}
$$

\section{APPENDIX B: AVERAGING SCHEME FOR MULTIPLE LOCAL MINIMA}

This section contains details on the approach we used to determine the artificial "temperature" for the Boltzmann weights as described in Sec. III A. We consider the situation that a set of local minima for which Eq. (12) becomes stationary is known. Let us specify by $\mathbf{a}_{y}(\phi)$ the vector of parameters $\left\{E_{\mu \nu}, \Gamma_{\mu \nu}^{(\kappa)}\right\}_{y}$ corresponding to one certain local minimum for a set of model parameters, labeled by $y$. In order to quantify the spectral weight distribution of the corresponding hybridization function $\underline{\Delta}_{\mathrm{aux}}\left[\omega ; \mathbf{a}_{y}(\phi)\right]$, we define

$$
\begin{aligned}
& m_{2}^{R}\left[\mathbf{a}_{y}(\phi)\right]=\int_{-\omega_{c}}^{\omega_{c}} \operatorname{Im}\left\{\Delta_{\text {aux }}^{R}\left[\omega ; \mathbf{a}_{y}(\phi)\right]\right\} \omega^{2} d \omega, \\
& m_{3}^{K}\left[\mathbf{a}_{y}(\phi)\right]=\int_{-\omega_{c}}^{\omega_{c}} \operatorname{Im}\left\{\Delta_{\text {aux }}^{K}\left[\omega ; \mathbf{a}_{y}(\phi)\right]\right\} \omega^{3} d \omega,
\end{aligned}
$$

which are similar to the second and third moments of $\Delta_{\text {aux }}^{R}$ and $\Delta_{\text {aux }}^{K}$, respectively. For the Keldysh component, a definition analogous to the first moment would yield the desired information as well but the choice above has been found to be more sensitive to details of $\Delta_{\text {aux }}^{K}$. The value of the corresponding cost function $\chi\left[\mathbf{a}_{y}(\phi)\right]$ of the $y$ th minimum is used as an artificial "energy" and enables one to define weights when making use of Boltzmann's statistic

$$
P_{y}(\phi, \beta)=\frac{1}{Z} e^{-\beta \chi\left[\mathbf{a}_{y}(\phi)\right]},
$$

where we introduced an artificial "temperature" $\beta^{-1}$. For each bias voltage separately, we are then able to calculate averaged quantities

$$
\overline{m_{2}^{R}}(\phi, \beta)=\sum_{y} P_{y}(\phi, \beta) m_{2}^{R}\left[\mathbf{a}_{y}(\phi)\right],
$$

as well as $\overline{m_{3}^{K}}(\phi, \beta)$ and $\bar{\chi}(\phi, \beta)$ in an analogous manner. The quantities $\overline{m_{2}^{R}}(\phi, \beta)$ and $\overline{m_{3}^{K}}(\phi, \beta)$ provide an estimate of the center of the spectral weight for the averaged set of hybridization functions for each bias voltage $\phi$.

Our goal is that these quantities vary in a smooth way when changing the bias voltage. To achieve this, we employ a minimum curvature scheme [133], meaning that we optimize the function

$$
\begin{aligned}
v_{c}(\beta)= & \int_{0}^{\phi_{\max }}\left\{w^{R}\left|\frac{\partial^{2}}{\partial \phi^{2}} \overline{m_{2}^{R}}(\phi, \beta)\right|^{2}+w^{K}\left|\frac{\partial^{2}}{\partial \phi^{2}} \overline{m_{3}^{K}}(\phi, \beta)\right|^{2}\right. \\
& \left.+w^{\chi}\left|\frac{\partial^{2}}{\partial \phi^{2}} \bar{\chi}(\phi, \beta)\right|^{2}\right\} d \phi,
\end{aligned}
$$

with respect to $\beta$. This determines the optimal artificial temperature, which ensures that the averaged cost function as well as the averaged spectral weight are as smooth functions of $\phi$ as possible, given the set of calculated minima $\left\{\mathbf{a}_{y}(\phi)\right\}$. As in many optimization problems, an arbitrariness exists in the definition of the quantities $\overline{m_{2}^{R}}(\phi, \beta)$ and $\overline{m_{3}^{K}}(\phi, \beta)$, as well as in choosing the values of the weights $w^{R}, w^{K}$, and $w^{\chi}$. In our case, all of the weights were chosen to be equal to one in units of $t$.

An improvement of the results, to a certain degree at least, could be expected when making use of extensions like a 
bias dependent $\beta(\phi)$. This has not been considered in this work since already a single variable $\beta$ provided quite smooth observables. As mentioned in the main text, in any case, it is obligatory to examine besides the averaged results also the ones for the absolute minima and/or for different averaging schemes, in order to avoid that physical discontinuities are averaged out. We stress that this approach has to be taken with due care since it is in some aspects arbitrary. However, it is useful to give an estimate of the error of the calculation, and can certainly identify regions in parameter space where the error is negligibly small.

\section{APPENDIX C: LINEAR CORRECTIONS}

In this section, we present a scheme to correct physical quantities up to linear order in the difference [165]

$$
\underline{D}(\omega)=\underline{\Delta}_{e x}(\omega)-\underline{\Delta}_{\text {aux }}(\omega)
$$

between the auxiliary and the exact hybridization functions. Although $\underline{D}(\omega)$ decreases rapidly with increasing number of auxiliary bath sites $N_{B}$, the size of the Hilbert space also increases exponentially with $N_{B}$. This poses a clear limit to the maximum value of $N_{B}$.

The idea is based on the fact that each physical quantity $O[\underline{\Delta}]$ is a functional of $\underline{\Delta}(\omega)$. Its exact value is, thus, obtained as $O\left[\underline{\Delta}_{e x}\right]$. For a finite $N_{B}$ there will always be a nonzero value of $\underline{D}(\omega)$ at some energies, so we will always obtain an approximate value $O\left[\underline{\Delta}_{\text {aux }}\right]$. A linear correction can be obtained by evaluating numerically the functional derivative of $O[\underline{\Delta}]$. Strictly speaking, considering that only $\operatorname{Im}\left[\Delta^{R}(\omega)\right]$ and $\operatorname{Im}\left[\Delta^{K}(\omega)\right]$ are independent functions, $O$ is a functional $O\left[\operatorname{Im}\left(\Delta^{R}\right), \operatorname{Im}\left(\Delta^{K}\right)\right]$. Suppose one knows the functional derivatives

$$
\frac{\delta O[\underline{\Delta}]}{\delta \operatorname{Im}\left[\Delta^{\alpha}(\omega)\right]}, \quad \alpha \in\{R, K\}
$$

then to linear order in $\underline{D}(\omega)$

$$
\begin{aligned}
O\left[\underline{\Delta}_{e x}\right] \approx & O\left[\underline{\Delta}_{\mathrm{aux}}\right] \\
& +\left.r \sum_{\alpha \in\{R, K\}} \int \frac{\delta O[\underline{\Delta}]}{\delta \operatorname{Im}\left[\Delta^{\alpha}\left(\omega_{0}\right)\right]}\right|_{\underline{\Delta}=\underline{\Delta}_{\mathrm{aux}}} \operatorname{Im}\left[D^{\alpha}\left(\omega_{0}\right)\right] d \omega_{0} \\
& +\mathcal{O}\left(\underline{D}^{2}\right),
\end{aligned}
$$

with $r=1$.

We evaluate the functional derivative numerically in the following way. One first evaluates $O\left[\underline{\Delta}_{\text {aux }}\right]$ at the optimum $\Delta_{\text {aux }}(\omega)$. Then, $O$ is evaluated at a "shifted" $\operatorname{Im}\left[\Delta^{\alpha}(\omega)\right]$, obtained by adding a delta function peaked around a certain energy $\omega_{0}$ :

$$
\delta_{\omega_{0}}(\omega) \equiv \delta\left(\omega-\omega_{0}\right)
$$

multiplied by a small coefficient $\epsilon$. The functional derivatives are then approximated linearly, by making use of the equations

$$
\begin{aligned}
& \frac{\delta O[\underline{\Delta}]}{\delta \operatorname{Im}\left[\Delta^{R}\left(\omega_{0}\right)\right]} \pm 2 \frac{\delta O[\underline{\Delta}]}{\delta \operatorname{Im}\left[\Delta^{K}\left(\omega_{0}\right)\right]} \\
& \approx \frac{1}{\epsilon}\left(O\left[\operatorname{Im}\left(\Delta^{R}\right), \operatorname{Im}\left(\Delta^{K}\right)\right]\right. \\
& \left.\quad-O\left[\operatorname{Im}\left(\Delta^{R}\right)-\epsilon \delta_{\omega_{0}}, \operatorname{Im}\left(\Delta^{K}\right) \mp 2 \epsilon \delta_{\omega_{0}}\right]\right),
\end{aligned}
$$

which become exact in the $\epsilon \rightarrow 0$ limit.

A (quasi)delta-peak correction $\epsilon \delta_{\omega_{0}}$ to $\Delta^{\alpha}(\omega)$ can be obtained by attaching an additional bath site $\left(N_{B}+1\right)$ with onsite energy $E_{N_{B}+1, N_{B}+1}=\omega_{0}$ directly to the impurity site with a hopping $E_{N_{B}+1, f}=\sqrt{\epsilon / \pi}$. The sum of $\Gamma_{N_{B}+1, N_{B}+1}^{(1)}$ and $\Gamma_{N_{B}+1, N_{B}+1}^{(2)}$ is proportional to the width of $\delta_{\omega_{0}}$ and, thus, should be taken as small as possible. In practice, one uses a discretization of the integration over $\omega_{0}$ in Eq. $(\mathrm{C} 1)$ and the width of the delta peaks has to be adjusted accordingly. Setting one of the components $\Gamma_{N_{B}+1, N_{B}+1}^{\kappa}$ to zero yields a peak in the Keldysh component with a coefficient $\pm 2 \epsilon$, respectively, as used in Eq. (C2).

Notice that the functional derivative (C2) amounts to carrying out two many-body calculations for each point $\omega_{0}$ on a system with $N_{B}+1$ bath sites. However, it is not necessary to repeat the calculation for each physical quantity of interest. In the linearly corrected current values presented in Sec. III B, a $\omega_{0}$ mesh of 200 points was used, whereby this number is likely to be reduced when optimizing the method.

Strictly speaking, the coefficient $r$ in Eq. (C1) should be 1 . However, for cases in which the linear correction is not small, this could produce an "overcorrection." In order to avoid this, we introduce a smaller ratio $r$ which is determined as follows: We evaluate the corrected self-energy at each $\omega$ via Eq. (C1) and $O=\underline{\Sigma}(\omega)$ with some value of $r<1$ and denote it $\underline{\Sigma}_{r}(\omega)$. We do the same for the Green's function of the auxiliary system and denote it $\underline{G}_{r}(\omega)$. Using Eqs. (5) and (6), we now have an estimate of an effective $r$-dependent auxiliary hybridization function of the linearly corrected system via

$$
\underline{\Delta}_{\mathrm{aux}, r}(\omega) \equiv \underline{g}_{0}^{-1}(\omega)-\underline{G}_{r}^{-1}(\omega)-\underline{\Sigma}_{r}(\omega) \text {. }
$$

In principle, for $r=1$ this gives $\underline{\Delta}_{e x}(\omega)$ up to $\mathcal{O}\left(\underline{D}^{2}\right)$. In practice, for finite $\underline{D}(\omega)$, one can introduce a cost function $\chi(r)$ analogous to Eq. (12) to minimize the difference $\mid \underline{\Delta}_{\text {aux }, r}(\omega)-$ $\underline{\Delta}_{e x}(\omega) \mid$ as a function of $r$. We checked that for the case in which the linear correction is a good approximation, the minimum occurs at $r=1$. If the minimum of $\chi(r)$ is situated at some value $r_{\min }<1$, then one corrects also other physical quantities according to Eq. (C1) with the same $r=r_{\min }$.

Alternatively to the correction ( $\mathrm{C} 1$ ) discussed above, one can use the numerical functional derivative evaluated via Eq. (C2) in order to estimate the sensitivity of the value of $O$ with respect to variations of $\operatorname{Im}\left[\Delta_{\text {aux }}^{\alpha}(\omega)\right]$ as a function of $\omega$ and $\alpha$. This is of use, in a second step, to adjust the weight function $W^{\alpha}(\omega)$ in Eq. (12), so that more sensitive $\omega$ regions acquire a larger weight.
[1] M. Hartmann, F. Brandão, and M. Plenio, Laser Photonics Rev. 2, 527 (2008).
[2] M. Raizen, C. Salomon, and Q. Niu, Phys. Today 50(7), 30 (1997). 
[3] D. Jaksch, C. Bruder, J. I. Cirac, C. W. Gardiner, and P. Zoller, Phys. Rev. Lett. 81, 3108 (1998).

[4] M. Greiner, O. Mandel, T. Esslinger, T. W. Hänsch, and I. Bloch, Nature (London) 415, 39 (2002).

[5] S. Trotzky, P. Cheinet, S. Fölling, M. Feld, U. Schnorrberger, A. M. Rey, A. Polkovnikov, E. A. Demler, M. D. Lukin, and I. Bloch, Science 319, 295 (2008).

[6] U. Schneider, L. Hackermuller, J. P. Ronzheimer, S. Will, S. Braun, T. Best, I. Bloch, E. Demler, S. Mandt, D. Rasch et al., Nat. Phys. 8, 213 (2012).

[7] S. Iwai, M. Ono, A. Maeda, H. Matsuzaki, H. Kishida, H. Okamoto, and Y. Tokura, Phys. Rev. Lett. 91, 057401 (2003).

[8] A. Cavalleri, T. Dekorsy, H. H. W. Chong, J. C. Kieffer, and R. W. Schoenlein, Phys. Rev. B 70, 161102 (2004).

[9] L. L. Bonilla and H. T. Grahn, Rep. Prog. Phys. 68, 577 (2005).

[10] I. Zutic, J. Fabian, and S. D. Sarma, Rev. Mod. Phys. 76, 323 (2004).

[11] G. Cuniberti, G. Fagas, and K. Richter, Introducing Molecular Electronics (Springer, Berlin, 2005).

[12] R. H. M. Smit, Y. Noat, C. Untiedt, N. D. Lang, M. C. van Hemert, and J. M. van Ruitenbeek, Nature (London) 419, 906 (2002).

[13] J. Park, A. N. Pasupathy, J. I. Goldsmith, C. Chang, Y. Yaish, J. R. Petta, M. Rinkoski, J. P. Sethna, H. D. Abruna, P. L. McEuen et al., Nature (London) 417, 722 (2002).

[14] W. Liang, M. P. Shores, M. Bockrath, J. R. Long, and H. Park, Nature (London) 417, 725 (2002).

[15] N. Agrait, A. L. Yeyati, and J. M. van Ruitenbeek, Phys. Rep. 377, 81 (2003).

[16] L. Venkataraman, J. E. Klare, C. Nuckolls, M. S. Hybertsen, and M. L. Steigerwald, Nature (London) 442, 904 (2006).

[17] D. Goldhaber-Gordon, J. Göres, M. A. Kastner, H. Shtrikman, D. Mahalu, and U. Meirav, Phys. Rev. Lett. 81, 5225 (1998).

[18] A. V. Kretinin, H. Shtrikman, and D. Mahalu, Phys. Rev. B 85, 201301 (2012).

[19] A. Mitra, S. Takei, Y. B. Kim, and A. J. Millis, Phys. Rev. Lett. 97, 236808 (2006).

[20] A. J. Leggett, S. Chakravarty, A. T. Dorsey, M. P. A. Fisher, A. Garg, and W. Zwerger, Rev. Mod. Phys. 59, 1 (1987).

[21] M. A. Cazalilla, Phys. Rev. Lett. 97, 156403 (2006).

[22] M. Rigol, V. Dunjko, and M. Olshanii, Nature (London) 452, 854 (2008).

[23] A. Nitzan and M. A. Ratner, Science 300, 1384 (2003).

[24] P. W. Anderson, Phys. Rev. 124, 41 (1961).

[25] J. Friedel, Can. J. Phys. 34, 1190 (1956).

[26] A. M. Clogston, B. T. Matthias, M. Peter, H. J. Williams, E. Corenzwit, and R. C. Sherwood, Phys. Rev. 125, 541 (1962).

[27] W. Brenig and K. Schönhammer, Z. Phys. 267, 201 (1974).

[28] A. Georges, G. Kotliar, W. Krauth, and M. J. Rozenberg, Rev. Mod. Phys. 68, 13 (1996).

[29] A. Cyril, The Kondo Problem to Heavy Fermions (Cambridge University Press, Cambridge, UK, 1997).

[30] D. Vollhardt, in Lecture Notes on the Physics of Strongly Correlated Systems, Vol. 1297 of AIP Conference Proceedings, edited by A. Avella and F. Mancini (AIP, New York, 2010), p. 339.

[31] W. Metzner and D. Vollhardt, Phys. Rev. Lett. 62, 324 (1989).

[32] J. Kondo, Prog. Theor. Phys. 32, 37 (1964).

[33] P. W. Anderson, J. Phys. C: Solid State Phys. 3, 2436 (1970).
[34] K. Yosida and K. Yamada, Prog. Theor. Phys. Suppl. 46, 244 (1970).

[35] K. Yamada, Prog. Theor. Phys. 53, 970 (1975).

[36] K. Yosida and K. Yamada, Prog. Theor. Phys. 53, 1286 (1975).

[37] K. Yamada, Prog. Theor. Phys. 54, 316 (1975).

[38] J. R. Schrieffer and P. A. Wolff, Phys. Rev. 149, 491 (1966).

[39] S. Y. Müller, M. Pletyukhov, D. Schuricht, and S. Andergassen, Phys. Rev. B 87, 245115 (2013).

[40] D. Bohr and P. Schmitteckert, Ann. Phys. (NY) 524, 199 (2012).

[41] L. H. Yu, Z. K. Keane, J. W. Ciszek, L. Cheng, J. M. Tour, T. Baruah, M. R. Pederson, and D. Natelson, Phys. Rev. Lett. 95, 256803 (2005).

[42] L. Tosi, P. Roura-Bas, and A. A. Aligia, J. Phys.: Condens. Matter 24, 365301 (2012).

[43] H. Prüser, M. Wenderoth, P. E. Dargel, A. Weismann, R. Peters, T. Pruschke, and R. G. Ulbrich, Nat. Phys. 7, 203 (2011).

[44] H. Aoki, N. Tsuji, M. Eckstein, M. Kollar, T. Oka, and W. Philipp, arXiv:1310.5329.

[45] P. Schmidt and H. Monien, arXiv:cond-mat/0202046.

[46] J. K. Freericks, V. M. Turkowski, and V. Zlatić, Phys. Rev. Lett. 97, 266408 (2006).

[47] J. K. Freericks, Phys. Rev. B 77, 075109 (2008).

[48] A. V. Joura, J. K. Freericks, and T. Pruschke, Phys. Rev. Lett. 101, 196401 (2008).

[49] M. Eckstein, M. Kollar, and P. Werner, Phys. Rev. Lett. 103, 056403 (2009).

[50] S. Okamoto, Phys. Rev. B 76, 035105 (2007).

[51] E. Arrigoni, M. Knap, and W. von der Linden, Phys. Rev. Lett. 110, 086403 (2013).

[52] P. Mehta and N. Andrei, Phys. Rev. Lett. 96, 216802 (2006).

[53] F. B. Anders, Phys. Rev. Lett. 101, 066804 (2008).

[54] F. B. Anders and S. Schmitt, J. Phys.: Conf. Ser. 220, 012021 (2010).

[55] A. Rosch, Eur. Phys. J. B 85, 6 (2012).

[56] Y. Meir, N. S. Wingreen, and P. A. Lee, Phys. Rev. Lett. 70, 2601 (1993).

[57] N. S. Wingreen and Y. Meir, Phys. Rev. B 49, 11040 (1994).

[58] T. Fujii and K. Ueda, Phys. Rev. B 68, 155310 (2003).

[59] H. Schoeller and G. Schön, Phys. Rev. B 50, 18436 (1994).

[60] S. Hershfield, J. H. Davies, and J. W. Wilkins, Phys. Rev. Lett. 67, 3720 (1991).

[61] H. Schoeller, Eur. Phys. J.: Spec. Top. 168, 179 (2009).

[62] A. Rosch, J. Paaske, J. Kroha, and P. Wölfle, J. Phys. Soc. Jpn. 74, 118 (2005).

[63] F. B. Anders and A. Schiller, Phys. Rev. B 74, 245113 (2006).

[64] D. Roosen, M. R. Wegewijs, and W. Hofstetter, Phys. Rev. Lett. 100, 087201 (2008).

[65] B. Doyon and N. Andrei, Phys. Rev. B 73, 245326 (2006).

[66] S. Weiss, J. Eckel, M. Thorwart, and R. Egger, Phys. Rev. B 77, 195316 (2008).

[67] F. B. Anders and A. Schiller, Phys. Rev. Lett. 95, 196801 (2005).

[68] M. Moeckel and S. Kehrein, Phys. Rev. Lett. 100, 175702 (2008).

[69] S. Kehrein, Phys. Rev. Lett. 95, 056602 (2005).

[70] G. Vidal, Phys. Rev. Lett. 93, 040502 (2004).

[71] S. R. White, Phys. Rev. B 48, 10345 (1993). 
[72] A. J. Daley, C. Kollath, U. Schollwöck, and G. Vidal, J. Stat. Mech. (2004) P04005.

[73] S. R. White and A. E. Feiguin, Phys. Rev. Lett. 93, 076401 (2004).

[74] U. Schollwoeck, Ann. Phys. (NY) 326, 96 (2011).

[75] P. Schmitteckert, Phys. Rev. B 70, 121302 (2004).

[76] F. Heidrich-Meisner, A. E. Feiguin, and E. Dagotto, Phys. Rev. B 79, 235336 (2009).

[77] M. Nuss, M. Ganahl, H. G. Evertz, E. Arrigoni, and W. von der Linden, Phys. Rev. B 88, 045132 (2013).

[78] M. Nuss, C. Heil, M. Ganahl, M. Knap, H. G. Evertz, E. Arrigoni, and W. von der Linden, Phys. Rev. B 86, 245119 (2012).

[79] M. Knap, W. von der Linden, and E. Arrigoni, Phys. Rev. B 84, 115145 (2011).

[80] F. Hofmann, M. Eckstein, E. Arrigoni, and M. Potthoff, Phys. Rev. B 88, 165124 (2013).

[81] C. Jung, A. Lieder, S. Brener, H. Hafermann, B. Baxevanis, A. Chudnovskiy, A. Rubtsov, M. Katsnelson, and A. Lichtenstein, Ann. Phys. (NY) 524, 49 (2012).

[82] R. Gezzi, T. Pruschke, and V. Meden, Phys. Rev. B 75, 045324 (2007).

[83] S. G. Jakobs, V. Meden, and H. Schoeller, Phys. Rev. Lett. 99, 150603 (2007).

[84] P. Werner, T. Oka, M. Eckstein, and A. J. Millis, Phys. Rev. B 81, 035108 (2010).

[85] G. Cohen, E. Gull, D. R. Reichman, and A. J. Millis, arXiv:1310.4151.

[86] J. E. Han, Phys. Rev. B 73, 125319 (2006).

[87] J. E. Han and R. J. Heary, Phys. Rev. Lett. 99, 236808 (2007).

[88] A. Dirks, P. Werner, M. Jarrell, and T. Pruschke, Phys. Rev. E 82, 026701 (2010).

[89] J. E. Han, A. Dirks, and T. Pruschke, Phys. Rev. B 86, 155130 (2012).

[90] A. Dirks, J. E. Han, M. Jarrell, and T. Pruschke, Phys. Rev. B 87, 235140 (2013).

[91] P. Dutt, J. Koch, J. Han, and K. Le Hur, Ann. Phys. (NY) 326, 2963 (2011).

[92] E. Muñoz, C. J. Bolech, and S. Kirchner, Phys. Rev. Lett. 110, 016601 (2013).

[93] A. M. Uimonen, E. Khosravi, A. Stan, G. Stefanucci, S. Kurth, R. van Leeuwen, and E. K. U. Gross, Phys. Rev. B 84, 115103 (2011).

[94] S. Smirnov and M. Grifoni, Phys. Rev. B 84, 125303 (2011).

[95] H. Schoeller and J. König, Phys. Rev. Lett. 84, 3686 (2000).

[96] M. Schiro and M. Fabrizio, Phys. Rev. Lett. 105, 076401 (2010).

[97] C. Timm, Phys. Rev. B 77, 195416 (2008).

[98] J. Eckel, F. Heidrich-Meisner, S. G. Jakobs, M. Thorwart, M. Pletyukhov, and R. Egger, New J. Phys. 12, 043042 (2010).

[99] S. Andergassen, V. Meden, H. Schoeller, J. Splettstoesser, and M. R. Wegewijs, Nanotechnology 21, 272001 (2010).

[100] L. D. Contreras-Pulido, J. Splettstoesser, M. Governale, J. König, and M. Büttiker, Phys. Rev. B 85, 075301 (2012).

[101] L. M. Falicov and J. C. Kimball, Phys. Rev. Lett. 22, 997 (1969).

[102] M. Eckstein and M. Kollar, Phys. Rev. Lett. 100, 120404 (2008).

[103] M. Eckstein, M. Kollar, and P. Werner, Phys. Rev. B 81, 115131 (2010).
[104] S. Okamoto, Phys. Rev. Lett. 101, 116807 (2008).

[105] C. Aron, G. Kotliar, and C. Weber, Phys. Rev. Lett. 108, 086401 (2012).

[106] C. Gramsch, K. Balzer, M. Eckstein, and M. Kollar, Phys. Rev. B 88, 235106 (2013).

[107] H. J. Carmichael, Statistical Methods in Quantum Optics: Master Equations and Fokker-Planck Equations, Vol. 1 of Texts and Monographs in Physics (Springer, Singapore, 2002).

[108] In our convention, lowercase $g$ denotes Green's functions of the system where the impurity is disconnected from the reservoirs, while capital $G$ denotes Green's functions of the connected system.

[109] Conventions for branch cuts are such that $g^{R}$ is causal.

[110] E. N. Economou, Green's Functions in Quantum Physics (Springer, Heidelberg, 2006).

[111] Note that in the present formalism, temperature would enter through the hybridization function $\Delta^{K}(\omega)$ only.

[112] This is in general true unless the system has bound states.

[113] L. P. Kadanoff and G. Baym, Quantum Statistical Mechanics: Green's Function Methods in Equilibrium and Nonequilibrium Problems (Addison-Wesley, Redwood City, CA, 1962).

[114] J. Schwinger, J. Math. Phys. 2, 407 (1961).

[115] L. V. Keldysh, Zh. Eksp. Teor. Fiz. 47, 1515 (1965) [JETP 20, 1018 (1965)].

[116] H. Haug and A.-P. Jauho, Quantum Kinetics in Transport and Optics of Semiconductors (Springer, Heidelberg, 1998).

[117] J. Rammer and H. Smith, Rev. Mod. Phys. 58, 323 (1986).

[118] A. Kamenev, Field Theory of Non-Equilibrium Systems (Cambridge University Press, Cambridge, 2011).

[119] M. Caffarel and W. Krauth, Phys. Rev. Lett. 72, 1545 (1994).

[120] C. Lanczos, J. Res. Natl. Bur. Stand. 45, 255 (1951).

[121] See Ref. [87].

[122] H.-P. Breuer and F. Petruccione, The Theory of Open Quantum Systems (Oxford University Press, Oxford, England, 2009).

[123] Operators are denoted by a hat $\hat{o}$, while superoperators acting on operators are denoted by a double hat $\hat{\hat{o}}$. For elementary fermionic creation/annihilation operators, we omit the hat. Finally, we use boldface for matrices and vectors in orbital indices.

[124] Alternatively, one could use the "star" representation, in which only diagonal and $E_{f, v}$ terms are nonzero.

[125] T. Prosen, New J. Phys. 10, 043026 (2008).

[126] A. A. Dzhioev and D. S. Kosov, J. Chem. Phys. 134, 044121 (2011).

[127] M. Schmutz, Z. Phys. B 30, 97 (1978).

[128] U. Harbola and S. Mukamel, Phys. Rep. 465, 191 (2008).

[129] From now on, we will omit the spin index, unless necessary.

[130] In our convention, $G_{\mu \nu}^{>+}(t)$ and $G_{\mu \nu}^{<-}(t)$ are zero for $t<0$, and vice versa.

[131] Notice that $\boldsymbol{D}$ commutes with $\lambda$, so $\frac{\boldsymbol{D}}{\omega-i \lambda}$ is well defined.

[132] D. F. Shanno, Math. Comp. 24, 647 (1970).

[133] W. H. Press, S. A. Teukolsky, W. T. Vetterling, and B. P. Flannery, Numerical Recipes 3rd Edition: The Art of Scientific Computing (Cambridge University Press, Cambridge, UK, 2007).

[134] J. D. Jackson, Classical Electrodynamics, 2nd ed. (Wiley, New York, 1975).

[135] For the particle-hole-symmetric model, the auxiliary system onsite energies are restricted to $E_{f f}=-\frac{U}{2}$ and $E_{\mu \mu}=$ $-E_{N_{B}+1-\mu, N_{B}+1-\mu}$ for $\mu \neq f$ as well as nearest-neighbor 
$\langle\ldots\rangle$ hopping to $E_{\langle\mu \nu\rangle}=(-1)^{\mu+v+1} E_{\left\langle N_{B}+1-\mu, N_{B}+1-v\right\rangle}$ while the dissipation matrices have to fulfill $\Gamma_{\mu \nu}^{(1)}=$ $(-1)^{\mu+\nu} \Gamma_{N_{B}+1-\mu, N_{B}+1-v}^{(2)}$.

[136] Y. Meir and N. S. Wingreen, Phys. Rev. Lett. 68, 2512 (1992).

[137] A.-P. Jauho, https://nanohub.org/resources/1877.

[138] J. W. Negele and H. Orland, Quantum Many-Particle Systems, Vol. 68 of Frontiers in Physics (Addison-Wesley, Redwood City, Calif., 1988).

[139] S. De Franceschi, R. Hanson, W. G. van der Wiel, J. M. Elzerman, J. J. Wijpkema, T. Fujisawa, S. Tarucha, and L. P. Kouwenhoven, Phys. Rev. Lett. 89, 156801 (2002).

[140] R. Leturcq, L. Schmid, K. Ensslin, Y. Meir, D. C. Driscoll, and A. C. Gossard, Phys. Rev. Lett. 95, 126603 (2005).

[141] J. S. Langer and V. Ambegaokar, Phys. Rev. 121, 1090 (1961).

[142] D. C. Langreth, Phys. Rev. 150, 516 (1966).

[143] L. Mühlbacher, D. F. Urban, and A. Komnik, Phys. Rev. B 83, 075107 (2011).

[144] J. König, J. Schmid, H. Schoeller, and G. Schön, Phys. Rev. B 54, 16820 (1996).

[145] A. Rosch, J. Paaske, J. Kroha, and P. Wölfle, Phys. Rev. Lett. 90, 076804 (2003).

[146] J. Dalibard, Y. Castin, and K. Mølmer, Phys. Rev. Lett. 68, 580 (1992).

[147] A. J. Daley, J. M. Taylor, S. Diehl, M. Baranov, and P. Zoller, Phys. Rev. Lett. 102, 040402 (2009).

[148] T. Prosen and M. Znidaric, J. Stat. Mech. (2009) P02035.

[149] A. Alvermann and H. Fehske, Phys. Rev. Lett. 102, 150601 (2009).

[150] A. Weisse, G. Wellein, A. Alvermann, and H. Fehske, Rev. Mod. Phys. 78, 275 (2006).

[151] C. D. Sherrill and H. F. Schaefer III, Adv. Quantum Chem. 34, 143 (1999).
[152] Y. Saad, Numerical Methods for Large Eigenvalue Problems, Revised Edition (Society for Industrial and Applied Mathematics, Philadelphia, 2011).

[153] P. Arbenz, Lecture Notes on Solving Large Scale Eigenvalue Problems, http://people.inf.ethz.ch/arbenz/ewp/lnotes. html.

[154] Z. Bai, J. Demmel, J. Dongarra, A. Ruhe, and H. van der Vorst, Templates for the Solution of Algebraic Eigenvalue Problems: A Practical Guide (Software, Environments and Tools) (Society for Industrial and Applied Mathematics, Philadelphia, 1987).

[155] M. Knap, E. Arrigoni, W. von der Linden, and J. H. Cole, Phys. Rev. A 83, 023821 (2011).

[156] Y. Saad, Iterative Methods for Sparse Linear Systems, 2nd ed. (Society for Industrial and Applied Mathematics, Philadelphia, 2003).

[157] R. Barrett, M. Berry, T. F. Chan, J. Demmel, J. Donato, J. Dongarra, V. Eijkhout, R. Pozo, C. Romine, and H. V. der Vorst, Templates for the Solution of Linear Systems: Building Blocks for Iterative Methods, 2nd ed. (SIAM, Philadelphia, PA, 1994).

[158] T. J. Park and J. C. Light, J. Chem. Phys. 85, 5870 (1986).

[159] Y. B. Bazaliy, E. Demler, and S.-C. Zhang, Phys. Rev. Lett. 79, 1921 (1997).

[160] M. H. Gutknecht, The unsymmetric Lanczos algorithms and their relations to Pade approximation, continued fractions, and the qd algorithm, http://www.math.ethz.ch/ mhg/.

[161] M. H. Gutknecht, Lanczos-type solvers for non-Hermitian linear systems, http://www.math.ethz.ch/ mhg/.

[162] R. Freund, M. Gutknecht, and N. Nachtigal, SIAM J. Sci. Comput. 14, 137 (1993).

[163] B. N. Parlett, D. R. Taylor, and Z. A. Liu, Math. Comp. 44, 105 (1985).

[164] H.-G. Weikert, H.-D. Meyer, L. S. Cederbaum, and F. Tarantelli, J. Chem. Phys. 104, 7122 (1996).

[165] For the sake of clarity, we specifically introduce the subscript ex to denote the exact hybridization function $\underline{\Delta}_{e x}$. This will be used only in this section. 